\title{
¿Puede el concepto "capacidad de cambio" de las arcillas, ser integramente aplicable a las puzolanas?
}

\author{
Is the clay "exchange capacity" concept \\ wholly applicable to pozzolans?
}

\begin{abstract}
R. TALERO
Instituto de Ciencias de la Construcción Eduardo Torroja (CSIC)

Correspondencia autor/Corresponding author: rtalero(ä;ictcc.csic.es

Fecha de recepcion: 27-111-03

Fecha de aceptación: 2-IV-()4

MADRID

\section{RESUMEN}

Una de las propiedades más caracteristicas de las arcillas es su "capacidad de cambio catiónico", o simplemente su "capacidad de camhio". Mediante este trabajo se ha logrado demostrar que algunas puzolanas naturales y artificiales, participan, en mayor o menor medida, y por diferentes causas, de esa caracteristica, si bien. de una forma parcial. Ello es dehido a que, a diferencia de las arcillas, su estado fisico-quimico original, vitreo vio amorfo en su mayor parte, se puede ver afectado por lo comin durante su hidratación con cemento Portland hasta llegar

SUMMARY

One of the most characteristic properties of clays is their "cation exchange capacity", otherwise known as their "exchange capacity". The research reported in this article shows that for various reasons some natural and artificial pozzolans exhibit this property. Ion exchange is only partial in the latter; hovever, because contrary to clays, on hydration with Portland cement, pozzolans may be nearly entirely converted from their mainly vitreous and/or amorphous original state into a variety of new more or less crystalline compounds.
\end{abstract} incluso a desaparecer practicamente, al transformarse en miltiples y variados compuestos más o menos cristalinos milevos.

Para ello se utilizaron 142 cementos de mezcla, 8 industriales, y el resto. 134, preparados en el laboratorio mediante mezcla de cementos Portland y. 3 puzolanas naturales (O. A y C) y artificiales (CV-19.CV-10 y M) en distintas proporciones porcentuales en masa.

Todos ellos se analizaron mediante el ensayo de Frattini a las edades de 7 y/o 28 dias, habiéndose determinado en su fase liquida correspondiente, no sólo sus contenidos de [OH] y [CaO], sino también sus correspondientes contenidos porcentuales de $\mathrm{Na}^{\prime}$ y $\mathrm{K}^{\prime}$, expresados como $\mathrm{Na}, \mathrm{O}$ . $K, O$, respectivamente: annque estos illtimos inicamente se le determinaron a los 4.5 cementos de mezcla más significativos para el objetivo de este trabajo. Por ialtimo. las tres puzolanas naturales turieron que ser analizadas por DRX para conocer también su composicion mineralógica.

Los resultados obtenidos han confirmado el objetivo que se pretendia demostrar; asi como también, que én tanto en cuanto una puzolana posea mavores contenidos de elementos alcalinos $N a$ yo $K$. malores posibilidade's tendrá de poder
For this purpose a total of 142 types of cement were tested in this study: 8 industrial and 1.34 laboratory cements, the latter prepared by mixing Portland cements with different proportions, by weight, of three natural and three artificial pozzolans.

All these cements were analysed by the Frattini test, 7 andior 28 days after mixing. Both [OH-] and $[\mathrm{CaO}]$ and the percentage content of $\mathrm{Na}^{-}$and $\mathrm{K}^{+}$. expressed as $\mathrm{Na}, \mathrm{O}$ and $K, O$. respectively, were determined in the liquid phase. The cation concentration was only determined for the 45 blended cements most relevant to the objective of the study. Finally, the three natural pozzolans were analyzed by XRD to identify their mineral composition.

The results obtained confirmed the working hypothesis. They also showed that the higher the content of $\mathrm{Na}$ and/or $\mathrm{K}^{\prime}$, the higher the likelihood of exchange capacity in the pozzolans. 
mostrar capacidad de cambio, en este caso con el catión Ca", principalmente, sin menoscabo de que su muy particular constitución mineralógica lo justifique también, caso de la zeolita y phillipsita en la puzolana C. lo que resultó ser, para ella, una causa adicional circunstancial muy importante. Pero no obstante y en cualquier caso, por el intercambio producido de iones $\mathrm{Na}$ y $\mathrm{K}^{\prime}$ por $\mathrm{Ca}^{2}{ }^{2}$, sus cementos de mezcla podrán cumplir más fäcilmente el ensayo de Frattini y los tiempos de firaguado, sobre todo,

- si el cemento con el que se meacle no es de elerada resistencia al ataque de los sulfatos, y

- si la proporción de mezcla ecemento Portland/Puzolana es la 60):40);

habicindose podido decir por tanto en un cierto sentido, que en tales circunstancias, el comportamiento de dicha puzolana C sobre todo, en dicho ensayo de Frattini, pareció ser más "aparente of ficticio que real". in this case primarily with Car. Nonetheless, the very special mineral composition of certain pozzolans, with minerals such as zeolite and phillipsite, may likewise contribute to this capacity. In any event, due to the cation exchange between $\mathrm{Na}^{*} / \mathrm{K}^{*}$ and $\mathrm{Ca}^{+}$, the blended cements involved met the Frattini test requirements more readily and had shorter setting times, especially:

- where the Portland cement with which they were mixed was not highly sulfate-resistant, and

- where the Portland cement/pozzolan ratio was 60/40.

Under these circumstances the behaviour of one of the pozzolans studied may, in one sense, be described to be "more apparent than real".
PALABRAS ClAVE: capacidad de cambio, arcillas, puzolanas, cementos Portland.

\section{INTRODUCCIÓN}

El concepto de capacidad de cambio nació como consecuencia del análisis y estudio detallado de la naturaleza físico-química de las arcillas. Por este motivo. es un concepto propio y característico de estos materiales.

Según este concepto, al estudiar físico-químicamente los cristales de arcilla, éstos suelen aparecer como una fase eminentemente cristalina que, al realizarle su análisis químico, demuestra poseer iones tales como H, Ca, Mg, K. Na, NH, Fe, Co, Cu, etc. Seguramente, unidos de forma débil al "núcleo ácido" o unidad fundamental de las arcillas.

El hecho se explicaría del siguiente modo: los cristales de arcilla suelen estar rodeados por una corona de cargas negativas, a lo que se debe que sean atraídos hacia el polo positivo, + , cuando se hace pasar una corriente eléctrica a través de sus suspensiones acuosas. Estas cargas negativas hacen que las arcillas se comporten como un ácido débil. La Figura 1 muestra de manera esquemática, como sería este comportamiento. Así, en la Figura la, se muestra el nícleo ácido de las arcillas con sus cargas negativas, -, en potencia, en la Figura $l b$ se pueden ver cargas negativas "ncutralizadas" por protones, $\mathrm{H}$, y por tanto con reacción francamente ácida, en la Figura $1 \mathrm{c}$ se puede ver sólo una parte saturada con $\mathrm{H}$, y otra parte, con cationes metálicos ó con el catión $\mathrm{NH}_{4}{ }^{\prime}, y$, por último, en la ligura $1 \mathrm{~d}$. se puede ver como todas las cargas están saturadas con cationes alcalinizantes.
KEYWORDS: exchange capacity, clays, pozzolans, Portland cements.

\section{INTRODUCTION}

Exchange capacity was first described on the occasion of in-depth study and analysis of the physical-chemical nature of clays and is regarded to be a concept characteristic of these materials.

In keeping with this concept, the chemical analysis of the crystalline phase of clay reveals the presence of ions such as $\mathrm{H}, \mathrm{Ca}, \mathrm{Mg}, \mathrm{K}, \mathrm{Na}, \mathrm{NH}_{4}, \mathrm{Fe}, \mathrm{Co}, \mathrm{Cu}$, etc., probably weakly bonded to the "acid core" or fundamental unit of the clay.

The explanation is that since clay crystals are usually surrounded by a ring of negative charges, they are attracted to the positive pole when an electric current is established in their aqueous scatters. Such negative charges make clays behave like weak acids, as shown schematically in Figure I. Figurel a, shows the clay acid core with its potentially negative charges, Figure 1 b, in turn, shows the negative charges "neutralised" by protons, $H$, i.e., a clearly acidic reaction, Figure I c shows some of the charges saturated with $H^{\prime}$, and some with metal cations or $\mathrm{NH}_{4}$ ' and, finally Figure $1 \mathrm{~d}$, shows all the charges saturated with alkaline cations. 


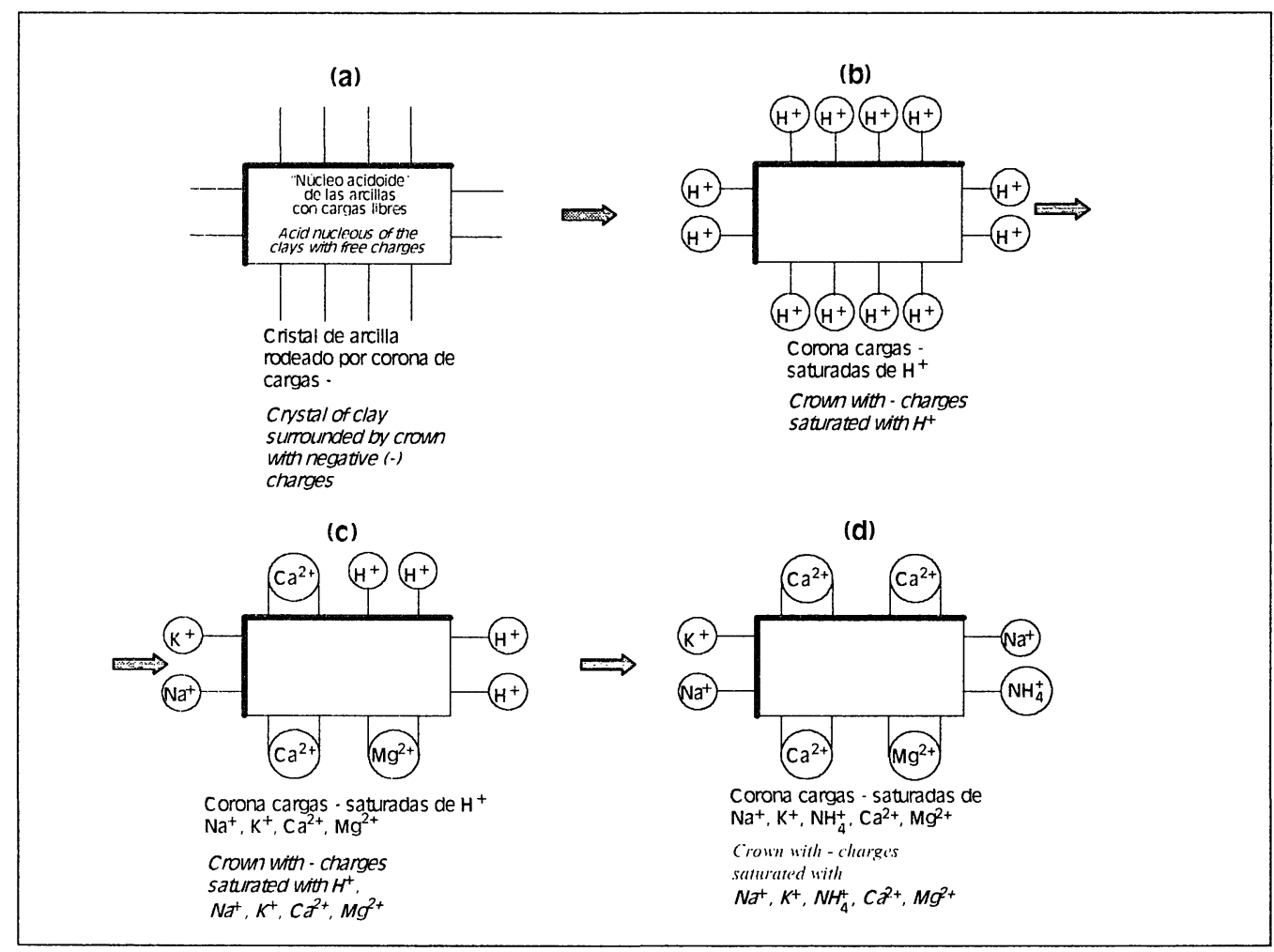

Figura 1.- Escluema del núcleo "ácido" de una arcilla (a), cuyas cargas negativas están siendo "neutralizadas" progresivamente por protones (b), y/o por cationes metálicos (c), yio por cationes alcalinos (d).

ligure l.- Sketch of the "acid" "ore of a clav (a). whose negative charges are being progressively "neutralized" by protons (b). and/or metalic cations (c), and'or cations which alkalinize (d).

Los cationes adheridos al "núcleo ácido" de algunas arcillas, se pueden cambiar a voluntad sin más que someterlas al contacto íntimo de una disolución acuosa más o menos concentrada de un catión dado, como es el caso de las permutitas, bajo la acción del CiNa.

No obstante y por lo general, la permuta no suele ser total en la realidad. Dado que la capacidad de cada ión para reemplazar a otro, depende de tres factores:

(a) De la concentración relativa ó número de iones (esto es una consecuencia de la Ley de Masas),

(b) De la earga de los iones, y

(c) De la velocidad de movimiento de cada ión (esto está relacionado con el tamaño de cada ión una vez solvatado) y su tiempo de contacto.

En definitiva y según todo lo anterior. la capacidad de cambio de una arcilla se podría definir como "el número total de valencias disponibles para retener cationes cambiables".

De acuerdo con dicha definición, la capacidad de cambio, basada principalmente en su "núcleo ácido", podría aplicarse para el caso de las puzolanas, ya sean éstas naturales o artificiales. Pues su intrínseca
The cations adhering to the acid core of some clays can be changed at will by: simply placing them in a more or less concentrated aqueous solution of another cation: $\mathrm{NaCl}$ with permutites, for instance.

Total exchange does not usually take place, however; because the capacity of each ion to replace the other depends on three factors:

(a) The relative concentration or number of ions (law of mass action),

(b) The charge of the ions, and

(c) The speed of ion motion (this is associated with the actual size of each ion after solvation) and the time of contact.

In light of the above, the exchange capacity of a clay can be defined as "the total number of valences available to retain exchangeable cations".

Thus defined, the exchange capacity concept, based primarily on the acid core of clays, is applicable to both natural and artificial pozzolans. The intrinsic activity of pozzolans, expressed in terms of their ability 
actividad puzolánica -representada por su poder de fijación de cationes $\mathrm{Ca}^{2-}$ del $\mathrm{Ca}(\mathrm{OH})$, de cualquier origen-, reside. sobre todo, en el también núcleo ácido de las mismas, el cual habrá de ser más ácido aún que el de las propias arcillas, al estar constituido en su mayor parte. por diferentes cantidades de sílice reactiva, $\mathrm{SiO}_{2}{ }^{\mathrm{r}-}(1)$, alúmina reactiva, $\mathrm{Al}_{2} \mathrm{O}_{3}{ }^{\mathrm{r}-}$ o alúmina tetra- o penta-coordinada( 1 ) -vítreas $y / 0$ amorfas- $y$ algo quizás también de óxido de Fe III reactivo, $\mathrm{Fe}_{2} \mathrm{O}_{3}{ }^{\mathrm{r}}$ -vítreo yio amorfo, pricipalmente-. Por otra parte, mientras al cristal de cualquier arcilla no le afecta ninguna de las sales que habitualmente se utilizan para determinarle su capacidad de cambio, a la fase mayoritaria, eminentemente vítrea y/o amorfa de las puzolanas, sí les puede afectar. Máxime, si alguna de dichas sales origina por hidrólisis disoluciones alcalinas. Ya que en un medio básico y cuanto más básico mejor, caso del $\mathrm{Ca}(\mathrm{OH})_{2}$. el $\mathrm{Na}(\mathrm{OH})$, el $\mathrm{K}(\mathrm{OH})$, etc., todas las puzolanas acaban siempre transformándose en diversos productos de reacción cuyo estado físico y composición química final son muy diferentes al de las puzolanas que los generó en cada caso -cristalinos como el $\mathrm{C}_{.+} \mathrm{AH}_{13}$, la ettringita de rápida formación, ett-rf (2-7), o, en su caso, la Sal de Friedel, de rápida formación también. sf-rf (8-10), o amorfos como el gel CSH, que podrían acabar siendo cristalinos con el tiempo, caso de las tobermoritas (11)-. Ello justificaría, por tanto, la imposibilidad de medir su capacidad de cambio como a las arcillas, si bien se podría confirmar si se produce durante el proceso de hidratación de determinadas puzolanas con cementos Portland, lo cual ha sido el objetivo principal de esta investigación.

Por otra parte, conviene recordar aquí también, que el poder de remplazo catiónico, a igualdad de concentración, sigue este orden: $\mathrm{H}^{-}>\mathrm{Ca}^{2+}>\mathrm{Mg}^{2-}>\mathrm{K}$ $>\mathrm{Na}^{\prime}>\mathrm{NH}_{4}$, estando el mismo más directamente relacionado con su carga y volumen resultante una vez solvatado cl catión (series liófilas o liotropas) (12), que con su volumen atómico. Así y según ambas series, el ión $\mathrm{K}$ 'solvatado desplazará al ión $\mathrm{Na}$ ' solvatado, mientras que el ión $\mathrm{NH}_{4}$ solvatado desplazaría al ión $\mathrm{Ba}^{2 \cdot}$ solvatado. Por contra, si la carga negativa de la lámina externa del "núcleo ácido" es muy fuerte, se puede llegar a producir una deshidratación del catión solvatado, y se pegará más al "núcleo ácido" el de menor radio y mayor carga(12). Pero, en cualquier caso, la referida actividad de una puzolana podría verse menoscabada por otros cationes solvatados más apropiados, diferentes al $\mathrm{Ca}^{2}$, que, por lo común, también se encuentran presentes, en mayor o menor proporción (dentro de su muy pequeño rango de variabilidad posible), en la fase liquida donde se producen las reacciones de hidratación de los cementos con y sin adiciones puzolánicas, como así ocurre con el $\mathrm{Na}$ y el K' to fix the $\mathrm{Ca}^{2}$ cations from $\mathrm{Ca}(\mathrm{OH})_{2}$ of any origin, lies chicfly in their acid core. This core should be more acidic than the core in clays since it consists primarily of different amounts of vitreous andior amorphous reactive silica, $\mathrm{SiO}_{2}^{{ }^{r}}(1)$, vitreous andior amorphous reactive alumina, $\mathrm{Al}_{2} \mathrm{O}_{3}^{--(1)}$, or tetra- or pentahedrallyco-ordinated alumina (1), perhaps some vitreous and or amorphous reactive $\mathrm{Fe} I \mathrm{II}$ oxide, $\mathrm{Fe}_{2} \mathrm{O}_{3}{ }^{*}$, as well as some minor crystalline phases. Moreover; whereas clay crystals are not affected by any of the salts generally. used to determine their exchange capacity, the eminently vitreous or amorphous majority phase of pozzolans can be impacted by these salts, particularly if they are the result of the hydrolysis of alkaline solutions. The reason is that in basic media and particularly in very basic media such as $\mathrm{Ca}(\mathrm{OH})$, $\mathrm{Na}(\mathrm{OH}), \mathrm{K}(\mathrm{OH})$, etc., all pozzolans are eventually converted into reaction products whose chemical composition and physical state differs from the chemistry and physical properties of the original substances. These products may be crystalline, such as $C_{4} A_{13}$ (rapid formation) ettringite ett-rf (2-7) or (rapid formation) Friedel's salt, sf-rf (8-10); or amorphous, such as CSH gel, which may nonetheless eventually crystallise, such as in the case of tobermorites (II). As a result, exchange capacity cannot be measured in pozzolans the way it is in clay, although the presence or otherwise of the process during the hydration of certain pozzolans with Portland cements (PC) can be confirmed, which was the chief aim of the present study.

Another fact to be borne in mind in this context is that, concentration being equal, the various cations can be ranked in terms of replacement power as follows: $\mathrm{H}^{+}>\mathrm{Ca}^{2}>\mathrm{Mg}^{2+}>\mathrm{K}^{+}>\mathrm{Na}^{+}>\mathrm{NH}_{4}{ }^{+}$. This order is more directly related to ion charge and volume after solvation (lyophyllic or lyotropic series) (12) than to atomic number: Thus, according to the arrangement of cations in these two series, solvated $K$ ions displace solvated $\mathrm{Na}$ ions, and solvated $\mathrm{NH}_{4}$ ions displace solvated $\mathrm{Ba}^{2 \cdot}$ ions. If the negative charge on the outer layer of the acid core is very strong, however, the solvated ion may become dehydrated and bond most strongly to the core with the smallest radius and highest charge (12). In any erent, pozzolan activity may be lessened by other more suitable solvated cations than C $a^{\prime *}$, which, like $N a$ and $K^{\prime}$, are usually present in some proportion (although with a very small range of variability) in the liquid phase in which cement hydration reactions take place, with and without pozzolanic additions. 
En definitiva, el concepto de capacidad de cambio es integramente aplicable a las arcillas, pero no tanto quizás a las puzolanas, las cuales y, en su caso, a lo sumo participarian de él sólo parcialmente, que es lo que se trata de demostrar mediante esta investigación.

\section{OBJETIVO}

I a presente investigación ha tenido por objetivo tratar de precisar si el concepto de capacidad de cambio de las arcillas es integramente aplicable, o no, a las puzolanas. Y, en caso de que lo fuere en alguna medida, determinar también:

- las circunstancias en las que dicho concepto capacidad de cambio de las arcillas y el concepto de actividad puzolánica de las puzolanas. son compatibles, y - su trascendencia en el comportamiento de estas últimas en el ensayo de Frattini.

\section{PARTE EXPERIMENTAL}

Para poder demostrar el desplazamiento catiónico(12) que se produce en el proceso de hidratación de algunas puzolanas con cemento Portland, semejante al que se produce para la determinación de la capacidad de cambio de las arcillas. se prepararon un total de 142 cementos de mezcla. De todos ellos,

- 8 cementos de mezcla fueron puzolánicos industriales o tipo CEM IV(13), y

- 134 cementos fueron obtenidos por mezcla de 6 cementos Portland tipo CEM I (en adelante, P) o de 6 de tipo CEM I/SR (en adelante, PY), y de una puzolana.

Las puzolanas utilizadas fueron: D y $\mathrm{N}$ (tierra de diatomeas), O, A y C' (puzolanas naturales de Olot, Almagro y Canarias, respectivamente), CV-10 y CV-19 (cenizas volantes) y $\mathrm{M}$ (metacaolín que se obtuvo por calentamiento a $750{ }^{\circ} \mathrm{C}$ de un caolín impurificado con aproximadamente el $50 \%$ de cuarzo, el cual sería determinado por D'TG).

Los resultados del análisis químico de cada uno de los 12 cementos Portland (13-15), de los 8 cementos puzolánicos (13-15) y de las 8 puzolanas (16-18), se encuentran en las Tablas 1 y 2 , respectivamente, mientras que la designación y numeración de todos los cementos de mezcla preparados y ensayados se encuentran en la Tabla 3. I as relaciones porcentuales cemento Portland/Puzolana fueron, en genera,1 80/ $20,70 / 309$ y $60 / 40$. I as cantidades pesadas de cemento Portland y puzolana se homogeneizaron en túrbula de laboratorio durante 30 minutos. Antes de preparar cada cemento de mezcla, las
In short, the exchange capacity concept is totally: applicable to clays, but perhaps less so to pozzolans, in which the phenomenon takes place only partially at best, which is what the present study attempts to prove.

\section{OBJECTIVE}

The aim of the present study is to determine whether the concept of exchange capacity of clays is applicable to pozzolans and to what extent. If applicable, firther objectives include determining:

- the circumstances in which the concept "exchange capacity of clay" and the concept "pozzolanic activity" are compatible, and

- the implications this may have on the behaviour of pozzolans in the Frattini test.

\section{EXPERIMENTAL}

A total of 142 blended cements were prepared to show; among others, the cationic displacement (12) taking place during hydration of some pozzolans with Portland cement, similar to the displacement recorded and used to determine the exchange capacity of clays:

- 8 of the blended cements were commercial pozzolanic or CEM-IV type cements (13), and

- 134 cements were obtained by mixing each of six CEM-I Portland cements (hereafter; $P$ ) or six CEM I/SR cements (hereafter, PY), with one of the pozzolans.

The pozzolans used were: $D$ and $N$ (diatomaceous), $O$, $A$ and $C$ (natural pozzolans from Olot, Almagro and the Canary Islands, respectively), CVIO and CVI9 (fly ashes) and $M$ (metakaolin, which was prepared by calcinating kaolin -with a quartz content of around $50 \%$, as determined by DTG-at $750{ }^{\circ} \mathrm{C}$ ).

The results of the chemical analysis of the 12 Portland cements (13-15), the 8 pozzolanic cements (13-15) and the 8 pozzolans (16-18) are shown in Tables 1 and 2. The labelling and numbering of all the prepared blended cements are given in Table 3. The Portland cement/pozzolan ratios were 8(0/20, 70/30 or 60/40. After weighing, the Portland cement and pozzolan were blended in a laboratory: mixer for 30 minutes. Before preparing each blended cement, the natural pozzolans, $O, A$ and $C$, were ground to almost 
TABLA 1/TABLE 1

Composición química de los cementos Portland y de las puzolanas

Chemical composition of Portland cements and pozzolans

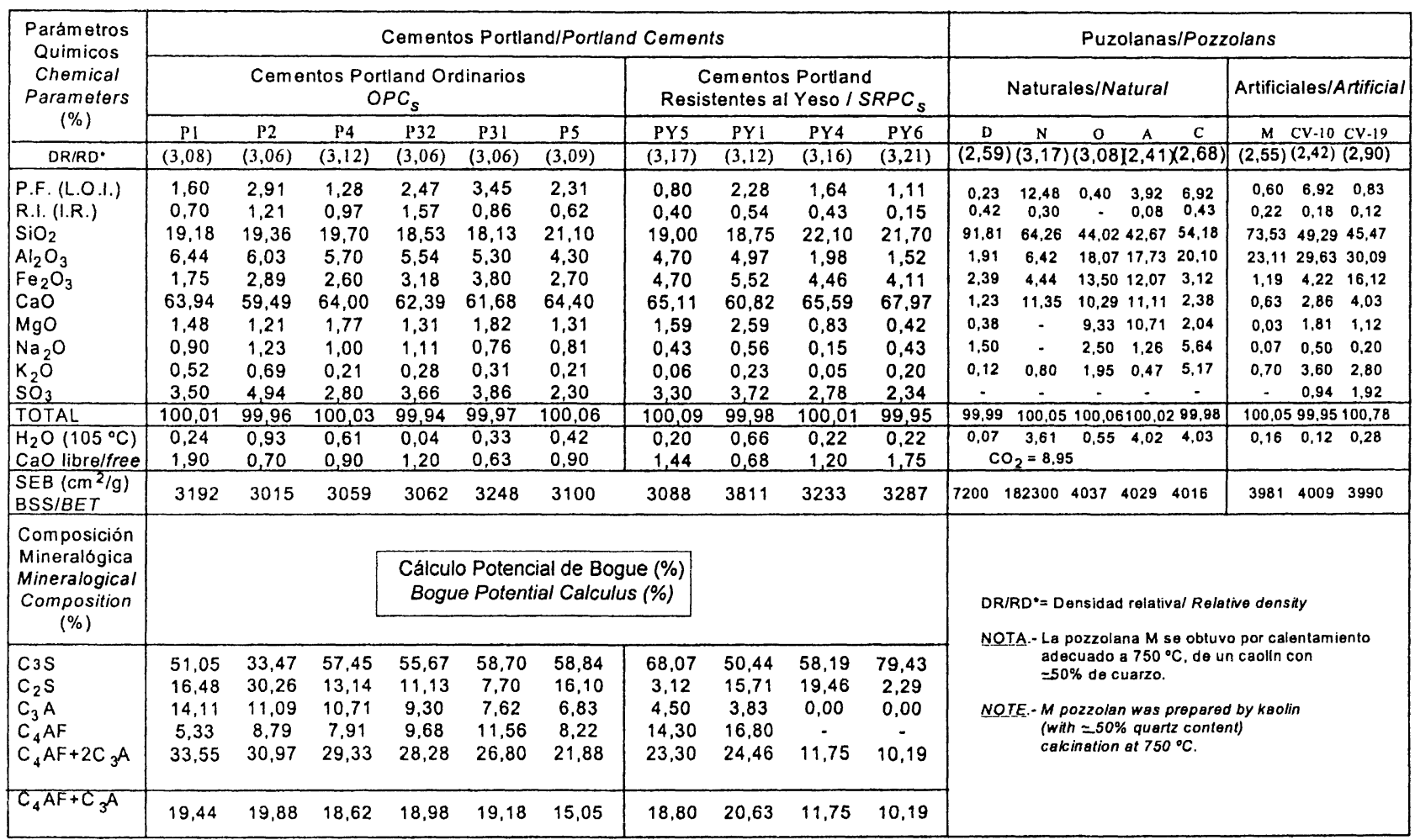

TABLA 2/TABLE 2

Composición química de los cementos de mezcla industriales Chemical composition of manufactured blended cements

\begin{tabular}{|c|c|c|c|c|c|c|c|c|}
\hline & \multicolumn{8}{|c|}{$\begin{array}{l}\text { Cementos de mezcla industriales } \\
\text { Manufactured blended cements }\end{array}$} \\
\hline & \multicolumn{8}{|c|}{ ASTM C 114-94 Standard (15) } \\
\hline & $\begin{array}{l}P O Z 1 \\
(3,02)\end{array}$ & $\begin{array}{l}\mathrm{PO} 22 \\
(3,09)\end{array}$ & $\begin{array}{l}\mathrm{POZ3} \\
(2,88) \\
\end{array}$ & $\begin{array}{l}\mathrm{POZ4} \\
(2,74) \\
\end{array}$ & $\begin{array}{l}P O Z 5 \\
(3,00)\end{array}$ & $\begin{array}{c}\text { POZ6 } \\
(2,92) \\
\end{array}$ & $\begin{array}{l}\mathrm{POZ7} \\
(2,91)\end{array}$ & $\begin{array}{l}\mathrm{POZ8} \\
(3.02) \\
\end{array}$ \\
\hline P.F. (L.O.I.) & 2.35 & 2,57 & 2,97 & 3,61 & 3,35 & 4,33 & 4,82 & 2,56 \\
\hline R.I. (I.R.) & - & 0,10 & 0,19 & 0.30 & 0.20 & 0,10 & 0,31 & 0.10 \\
\hline $\mathrm{SiO}_{2}$ & 23,85 & 24,01 & 28,23 & 34,21 & 22,29 & 23,81 & 26,90 & 23,74 \\
\hline $\mathrm{Al}_{2} \mathrm{O}_{3}$ & 7,25 & 7,91 & 8,96 & 11,80 & 8,68 & 9,18 & 7,54 & 8,96 \\
\hline $\mathrm{Fe}_{2} \mathrm{O}_{3}$ & 7,65 & 5,04 & 3,68 & 3,40 & 5.72 & 5,82 & 5,53 & 6,80 \\
\hline $\mathrm{Ca}$ & 52,02 & 52,37 & 51,20 & 40,71 & 53,95 & 47,67 & 47,49 & 49,16 \\
\hline $\mathrm{MgO}$ & 3,25 & 3,85 & 1,47 & 1,60 & 2,66 & 5,34 & 1.93 & 3.84 \\
\hline $\mathrm{Na}_{2} \mathrm{O}$ & 0,30 & 0,40 & 1,18 & 1,50 & 0,20 & 0,40 & 0,71 & 0,39 \\
\hline & 0,60 & 0,69 & 0,58 & 0,91 & 0,50 & 0,88 & 1.12 & 0,98 \\
\hline & 2,78 & 3,11 & 1,63 & 1,92 & 2,50 & 2,54 & 3,70 & 3,51 \\
\hline TOTAL & 100,05 & 100,05 & 100.09 & 99,96 & 100,05 & 100,07 & 100,05 & 100,04 \\
\hline $\mathrm{H}_{2} \mathrm{O}\left(105^{\circ} \mathrm{C}\right)$ & 1.37 & 1,36 & 1,62 & 2,23 & 0,97 & 2,34 & 1,26 & 1,05 \\
\hline CaO libre/free & 0,60 & 1.29 & 1,27 & 0.94 & 1.87 & 0,63 & 0,95 & 0,38 \\
\hline
\end{tabular}

puzolanas naturales $\mathrm{O}, \mathrm{A}$ y $\mathrm{C}$ se habían molido hasta dejar un residuo en el tamiz de $45 \mu \mathrm{m}$ de luz de malla $\leq 20,0 \%$ (19) (el residuo de las tres fue de aproximadamente $18,0 \%$ ). En cambio las dos cenizas volantes no tuvieron que ser molturadas, ya que dejaron un residuo en dicho tamiz $\leq 34,0 \%$ (20) (CV-19 dejó $29,0 \%$ y CV-10, $32,0 \%$ ) y las diatomitas $\mathrm{D}$ y $\mathrm{N}$ y el metakaolín $\mathrm{M}$, tampoco, ya que el residuo que dejaron estuvo comprendido entre el $5,0 \%$ y el $10,0 \%$, es decir, fue aún menor que el de las tres puzolanas naturales. entirely pass the $45 \mu \mathrm{m}$ mesh sieve (residue $\leq 20.0 \%$ ) (19) (the actual residue in all three cases was 18.0\%). The two fly ashes did not have to be ground, since they left a residue in the sieve $\leq 34.0 \%$ (20) (the actual values were, for $C V-1929.0 \%$ and for $C V-1032.0 \%)$. Neither the diatomites, $D$ and $N$, or metakaolin (M pozzolan), were ground, either, as they left a residue of from $5.0 \%$ to $10.0 \%$, i.e. even less than the three natural pozzolans after grinding. 
TABLA 3/TABLE 3

Ensayo de Frattini de los cementos de mezcla. Resultados a 7 y 28 días

Frattini test of the blended cements. Results at 7 and 28 days

\begin{tabular}{|c|c|c|c|c|c|c|c|c|c|c|c|c|c|c|c|c|c|c|c|c|}
\hline \multirow{2}{*}{ 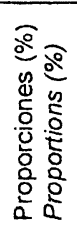 } & \multirow{2}{*}{ 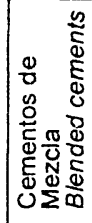 } & \multicolumn{2}{|c|}{$\mathrm{CaO}(\mathrm{mM} / \mathrm{l}$} & \multicolumn{2}{|c|}{$\mathrm{OH}^{-}(\mathrm{mM} / \mathrm{l})$} & \multirow{2}{*}{ 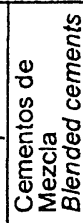 } & \multicolumn{2}{|c|}{$\mathrm{CaO}(\mathrm{mM} / \mathrm{l})$} & \multicolumn{2}{|c|}{$\mathrm{OH}^{-}(\mathrm{mM} / \mathrm{l})$} & \multirow{2}{*}{ 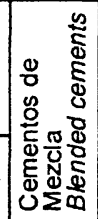 } & \multicolumn{2}{|c|}{$\mathrm{CaO}(\mathrm{mM} / \mathrm{l})$} & \multicolumn{2}{|c|}{$\mathrm{OH}^{-}(\mathrm{mM} / \mathrm{l})$} & \multirow{2}{*}{ 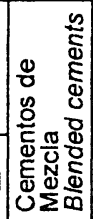 } & \multicolumn{2}{|c|}{$\mathrm{CaO}(\mathrm{mM} / \mathrm{I}$} & \multicolumn{2}{|c|}{ 1) $\mathrm{OH}^{-}(\mathrm{mM} / \mathrm{l})$} \\
\hline & & $7 d$ & $28 d$ & $7 \mathrm{~d}$ & $28 d$ & & $7 d$ & $28 d$ & $7 d$ & $28 d$ & & $7 d$ & $28 d$ & $7 d$ & $28 \mathrm{~d}$ & & $7 d$ & $28 d$ & $7 d$ & $28 \mathrm{~d}$ \\
\hline $80 / 20$ & $\mathrm{P} 1 / \mathrm{D}$ & 6,50 & & 90,02 & & $\mathrm{P} 1 / \mathrm{N}$ & 4,00 & & 41,50 & & $\mathrm{P}_{1 / M}$ & 5,00 & & 68,83 & & POZ1 & 10,63 & 6,10 & 64,46 & 669,85 \\
\hline $70 / 30$ & $\mathrm{P} 1 / \mathrm{D}$ & 5,45 & & 66,17 & & $\mathrm{P} 1 / \mathrm{N}$ & 3,05 & & 22,64 & & $\mathrm{P} 1 / \mathrm{M}$ & 2,40 & & 53,51 & & POZ2 & $|9,80|$ & 5,55 & 65,76 & d72,53 \\
\hline $60 / 40$ & $\mathrm{P} 1 / \mathrm{D}$ & 4,36 & & 42,25 & & $\mathrm{P} 1 / \mathrm{N}$ & 2,80 & & 10,56 & & $\mathrm{P} 1 / \mathrm{M}$ & 1,63 & & 28,76 & & POZ3 & $6,80 \mid$ & & 40,50 & \\
\hline $80 / 20$ & $\mathrm{P} 2 / \mathrm{D}$ & 9,48 & & 47,73 & & $\mathrm{P} 2 / \mathrm{N}$ & 7,80 & & 30,94 & & $\mathrm{P} 2 / \mathrm{M}$ & 4,95 & & 40,01 & & POZ4 & $1,00 \mid$ & & 78,05 & \\
\hline $70 / 30$ & $\mathrm{P} 2 / \mathrm{D}$ & 8,30 & & 45,22 & & $\mathrm{P} 2 / \mathrm{N}$ & 4,25 & & 11,57 & & $\mathrm{P} 2 / \mathrm{M}$ & 2,50 & & 30,76 & & POZ5 & 3,00 & & 92,82 & \\
\hline $60 / 40$ & $\mathrm{P} 2 / \mathrm{D}$ & 7,13 & & 42,71 & & $\mathrm{P} 2 / \mathrm{N}$ & 0,75 & & $\mid 11,07$ & & $\mathrm{P} 2 / \mathrm{M}$ & 1,80 & & 52,01 & & POZ6 & 5,65 & & 57,57 & \\
\hline $80 / 20$ & P32/D & 5,30 & & 69,60 & & $\mathrm{P} 32 / \mathrm{N}$ & 4,00 & & 44,77 & & P32/M & 5,14 & & 60,46 & & POZ7 & 10,50 & 8,65 & 52,07 & $\frac{1}{56} 6,50$ \\
\hline $70 / 30$ & P32/D & 4,50 & & 68,91 & & P32/N & 2,80 & & 27,16 & & P32/M & 3,50 & & 49,13 & & POZ8 & 4,93 & & 58,86 & \\
\hline $60 / 40$ & P32/D & 3,70 & & 68,08 & & P32/N & 1,90 & & 13,08 & & P $32 / M$ & 1,85 & & 37,41 & & & & & & \\
\hline $80 / 20$ & $\mathrm{P} 4 / \mathrm{D}$ & 7,75 & & 57,70 & & $\mathrm{P} 4 / \mathrm{N}$ & 5,75 & & 32,70 & & P4/M & 5,28 & & 57,26 & & & & & & \\
\hline $70 / 30$ & $\mathrm{P} 4 / \mathrm{D}$ & 7,45 & & 55,76 & & $\mathrm{P} 4 / \mathrm{N}$ & 3,65 & & 25,15 & & $\mathrm{P} 4 / \mathrm{M}$ & 4,15 & & 46,68 & & $\frac{P 31}{c V 10}$ & 0,85 & & 55,27 & \\
\hline $60 / 40$ & $\mathrm{P} 4 / \mathrm{D}$ & 6,59 & & 53,79 & & $\mathrm{P} 4 / \mathrm{N}$ & 3,60 & & 9,05 & & $\mathrm{P} 4 / \mathrm{M}$ & 1,68 & & 35,03 & & & & & & \\
\hline $80 / 20$ & P31/D & 4,55 & & 65,83 & & P31/N & 4,05 & & 48,29 & & P31/M & 5,25 & & 78,42 & & & & & & \\
\hline $70 / 30$ & P31/D & 3,55 & & 63,52 & & P31/N & 3,75 & & 40,24 & & P31/M & 3,15 & & $|60,22|$ & & $\left|\frac{P_{31}}{C V_{10}}\right|$ & 2,50 & & 55,03 & \\
\hline $60 / 40$ & P31/D & 2,15 & & 61,22 & & P31/N & 2,90 & & $\mid 11,57$ & & P31/M & 1,08 & & 42,01 & & & & & & \\
\hline $80 / 20$ & $P 5 / D$ & 11,72 & & 58,32 & & $\mathrm{P} 5 / \mathrm{N}$ & 6,40 & & 36,72 & & $\mathrm{P} 5 / \mathrm{M}$ & 9,10 & & 57,41 & & & & & & \\
\hline $70 / 30$ & $P 5 / D$ & 8,60 & & 51,41 & & $\mathrm{P} 5 / \mathrm{N}$ & 4,75 & & 7,55 & & P5/M & 2,05 & & 22,61 & & & & & & \\
\hline $60 / 40$ & $P 5 / D$ & 5,50 & & 44,56 & & $\mathrm{P} 5 / \mathrm{N}$ & 3,70 & & 5,05 & & $\mathrm{P} 5 / \mathrm{M}$ & 1,59 & & 30,01 & & & & & & \\
\hline $80 / 20$ & PY5/D & 7,35 & & 46,81 & & PY5/N & & & & & PY5/M & 5,02 & & 68,58 & & & & & & \\
\hline $70 / 30$ & PY5/D & 6,20 & & 46,27 & & PY5/N & & & & & PY5/M & 3,13 & & 46,81 & & & & & & \\
\hline $60 / 40$ & PY $5 / D$ & 5,05 & & 45,79 & & PY $5 / N \mid$ & & & & & PY5/M & 2,45 & & 24,95 & & & & & & \\
\hline $80 / 20$ & PY1/D & 8,88 & & 68,01 & & PY $1 / N$ & $\mid 15,00$ & & $\mid 16,53$ & & PY1/M & 8,05 & & 58,22 & & & & & & \\
\hline $70 / 30$ & PY1/D & 6,95 & & 54,01 & & $|\mathrm{PY} / \mathrm{N}|$ & $|11,40|$ & & 6,04 & & PY1/M & 5,25 & & 44,79 & & & & & & \\
\hline $60 / 40$ & PY1/D & 4,90 & & 40,01 & & PY1/N & 5,85 & & 2,52 & & PY1/M & 1,75 & & 32,51 & & & & & & \\
\hline $80 / 20$ & PY $4 / D$ & 17,50 & & 49,37 & & PY4/N & 17,15 & & 18,10 & & PY4/M & $\mid 10,15$ & & 38,02 & & & & & & \\
\hline $70 / 30$ & PY 4/D & 16,50 & & 44,61 & & PY $4 / N$ & 12,23 & & 8,05 & & PY4/M & 7,98 & & 29,01 & & & & & & \\
\hline $60 / 40$ & PY4/D & 12,80 & & 41,94 & & PY4/N & 9,25 & & 2,52 & & PY4/M & 4,30 & & 20,31 & & & & & & \\
\hline $80 / 20$ & PY6/D & 16,25 & & 47,41 & & PY6/N & 11,60 & & 21,63 & & PY6/M & $\mid 12,30$ & & 36,01 & & & & & & \\
\hline $70 / 30$ & PY6/D & 14,87 & & 45,01 & & PYG/N & 11,23 & & 14,08 & & PY6/M & $|9,00|$ & & 27,91 & & & & & & \\
\hline $60 / 40$ & PY6/D & 13,25 & & $\mid 41,40$ & & PY6/N & 9,95 & & 2,01 & & PY6/M & 5,32 & & 19,81 & & & & & & \\
\hline $80 / 20$ & $\mathrm{P} 1 / 0$ & 5,30 & 2,60 & 99,97 & 102,08 & $\mathrm{P} 1 / \mathrm{A}$ & 3,80 & & $|63,39|$ & & $\mathrm{P} 1 / \mathrm{C}$ & $|3,30|$ & & $\mid 82,24$ & & & & & & \\
\hline $70 / 30$ & $\mathrm{P} 1 / \mathrm{O}$ & 4,80 & 2,03 & 92,23 & 95,37 & $P 1 / A$ & 3,74 & & 67,42 & & $\mathrm{P} 1 / \mathrm{C}$ & 3,02 & & 84,06 & & & & & & \\
\hline $60 / 40$ & $\mathrm{P} 1 / \mathrm{O}$ & 3,45 & & 82,24 & & $\mathrm{P} 1 / \mathrm{A}$ & 3,65 & & 71,55 & & $\mathrm{P} 1 / \mathrm{C}$ & 2,81 & & 85,79 & & & & & & \\
\hline $80 / 20$ & $\mathrm{P} 2 / \mathrm{O}$ & 15,68 & 5,93 & 80,26 & 72,98 & $\mathrm{P} 2 / \mathrm{A}$ & 13,01 & 6,80 & 61,18 & 55,01 & 1) $P 2 / C$ & 4,10 & & 75,65 & & & & & & \\
\hline $70 / 30$ & $\mathrm{P} 2 / \mathrm{O}$ & 14,36 & 3,80 & 71,17 & 73,22 & $\mathrm{P} 2 / \mathrm{A}$ & 12,15 & 5,28 & 50,33 & 58,19 & $\mathrm{P} 2 / \mathrm{C}$ & 2,20 & & 80,52 & & & & & & \\
\hline $60 / 40$ & $\mathrm{P} 2 / \mathrm{O}$ & 13,20 & 1,17 & 62,01 & 73,42 & $P 2 / A$ & $\mid 11,63$ & & 40,01 & & $\mathrm{P} 2 / \mathrm{C}$ & $|1,50|$ & & 85,43 & & & & & & \\
\hline $80 / 20$ & P31/O & 14,05 & 5,75 & 86,33 & 75,01 & $\mid \mathrm{P} 31 / \mathrm{A}$ & 6,75 & & $|77,24|$ & & $\mathrm{P} 31 / \mathrm{C}$ & 2,55 & & 111,87 & & & & & & \\
\hline $70 / 30$ & P31/O & 13,20 & 4,25 & $|77,50|$ & 75,32 & $P 31 / A$ & 6,10 & & 69,48 & & $\mathrm{P} 31 / \mathrm{C}$ & 2,00 & & 91,80 & & & & & & \\
\hline $60 / 40$ & P31/O & 10,95 & 2,70 & 65,21 & 76,99 & $P 31 / A$ & 5,83 & & 60,76 & & $\mathrm{P} 31 / \mathrm{C}$ & 1,62 & & 80,02 & & & & & & \\
\hline $80 / 20$ & PY4/O & 14,90 & 10,50 & 49,01 & 20,21 & PY 4/A & 13,47 & 7,80 & 111,7 & 42,15 & $5 \mid \mathrm{PY} 4 / \mathrm{C}$ & 6,50 & & 67,41 & & & & & & \\
\hline $70 / 30$ & PY $4 / 0$ & 14,25 & 14,10 & 52,31 & 24,21 & PYAIA & 15,25 & 10,55 & 45,11 & 44,60 & $\mid P Y 4 / C$ & 3,90 & & $|77,22|$ & & & & & & \\
\hline $60 / 40$ & PY4/O & $\mid 12,25$ & & 28,21 & & PY $4 / A$ & $|13,40|$ & & 41,71 & & PY4/C & 3,21 & & $|93,20|$ & & & & & & \\
\hline $80 / 20$ & PY6/O & $\mid 16,65$ & 7,00 & 54,61 & 54,41 & PYG/A & $\mid 19,33$ & 11,68 & 53,22 & 32,01 & $\mid \mathrm{PY} 6 / \mathrm{C}$ & 7,35 & 4,88 & 60,02 & 73,22 & & & & & \\
\hline $70 / 30$ & PY6/O & 13,50 & 14,75 & 53,71 & 24,81 & PYG/A & $|16,60|$ & 10,85 & 48,01 & $|37,49|$ & $9 \mid \mathrm{PY} 6 / \mathrm{C}$ & 3,95 & & $\mid 67,21$ & & & & & & \\
\hline $60 / 40$ & PY6/O & 9,95 & & 50,91 & & PYG/A & 13,30 & 10,00 & 44,01 & 45,09 & PYG/C & 2,55 & & 81,42 & & & & & & \\
\hline \multicolumn{21}{|c|}{$\mathrm{d}=$ días $/$ days } \\
\hline & & & satur & $\begin{array}{l}\text { es de } \\
\text { ración }\end{array}$ & n (=re & $\begin{array}{l}\text { res en } \\
\text { esultad }\end{array}$ & $\begin{array}{l}\text { negrit } \\
(0+)\end{array}$ & & & & 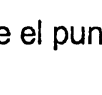 & & & & & & & & & \\
\hline & & $\begin{array}{l}\text { The } \\
\text { sub }\end{array}$ & satur & ration & regic & $\begin{array}{l}\text { s in bo } \\
\text { ion } 1=+\end{array}$ & resu & $\begin{array}{l}\text { pefac } \\
\text { att) }\end{array}$ & ce me & ent & that the & poin & $m$ & แI & & & & & & \\
\hline
\end{tabular}


Los cementos Portland, P-n ${ }^{\circ}$ y PY-n”, y las puzolanas, se seleccionaron de acuerdo con los siguientes criterios:

1. Los cementos Portland, P- $n^{\circ}$, deberían poseer contenidos apreciables de $\mathrm{Na}^{\prime}$ y $\mathrm{K}^{-}$(expresados como $\mathrm{Na}_{2} \mathrm{O}(\%)$ y $\mathrm{K}_{2} \mathrm{O}(\%)$, respectivamente), hasta el punto de que pudieran ser calificados de "elevados o nuy elevados contenidos de alcalinos"(21) (22), porque la suma $\left[\mathrm{Na}_{2} \mathrm{O}(\%)+\left(0,658 \times \mathrm{K}_{2} \mathrm{O}(\%)\right)\right]$ resultaba ser $>0,60 \%$ (21) (22): mientras que los de algunos cementos Portland PY, sus contenidos debían ser menos elevados hasta el punto de que la referida suma de cationes alcalinos tenía que ser necesariamente $\leq 0,60 \%$, para así poderlos calificar como de "no elevados contenidos de alcalinos"(21) (22), caso del PY-4 y el PY-6, véase la Tabla 1.

2. Las puzolanas naturales y artificiales, independientemente de su origen y contenido de factores hidráulicos, se seleccionaron de modo y manera que su contenido de alcalinos $\mathrm{Na}^{\prime \prime}$ y $\mathrm{K}^{+}$. expresados como $\left[\mathrm{Na}_{2} \mathrm{O}(\%)+\left(0,658 \times \mathrm{K}_{2} \mathrm{O}(\%)\right)\right]$, variara de forma gradativa e incluso extrema, de una a otra, con las condiciones adicionales de que,

- entre las de mayor y menor contenido existiera una notable diferencia, y que,

- algunas de ellas incumplieran, en mayor o menor medida, lo especificado al respecto como máximo contenido posible permitido de tales cationes alcalinos, . por la propuesta correspondiente de ASINEL (16) (idem $\mathrm{d} \leq 2,0 \%$ ), c incluso - por la norma ASTM C 618-94 a (20) (su contenido de alcalinos $\mathrm{Na}$ y $\mathrm{K}$, expresados como $\left[\mathrm{Na}_{2} \mathrm{O}(\%)+\right.$ $\left.\left.\left(0,658 \times \mathrm{K}_{2} \mathrm{O}(\%)\right)\right] \leq 1,50 \%\right)$.

A todos los cementos de mezcla anteriores se les determinó el Índice de Puzolanicidad mediante el ensayo de Frattini (14) (Tabla 3), a pesar de que este ensayo está especificado para los cementos puzolánicos o cementos CEM IV (13) únicamente. así como también, a algunos de ellos, sus Tiempos de Fraguado (13). Las cantidades de fase líquida del ensayo de Frattini. correspondientes a los cementos de mezcla de las puzolanas D y $\mathrm{N}$, principalmente, resultaron ser notablemente menores que las del resto de puzolanas ensayadas y comparadas con ellas y/o cualquier cemento puzolánico tradicional. De aquí que los resultados obtenidos de sus cementos de mezcla, tuvieran que ser determinados en partes alícuotas de fase líquida, menores que las especificadas para dicho ensayo. La razón habría que buscarla en su especial morfología por ser ambas diatomitas, pero, sobre todo, en su muy particular composición química al estar constituidas por sílice amorfa en casi su totalidad.
The criteria applied to select the Portland cements, $P$ No. and PY-No. and the pozzolans were as follows:

\section{The Portland cements, P-No., had to contain} sufficient amounts of $\mathrm{Na}^{+}$and $\mathrm{K}^{+}$(expressed as $\mathrm{Na}_{2} \mathrm{O}(\%)$ and $\mathrm{K}_{2} \mathrm{O}(\%)$, respectively) to be regarded to have "high or very high alkaline contents" (21) (22), with $[\mathrm{Na}, \mathrm{O}(\%)+(0.658 \times \mathrm{K}, \mathrm{O}(\%))]>0.60 \%(2 \mathrm{l})$ (22). Some of the PY Portland cements, in turn, were required to "not have high alkaline cation contents", i.e., in these cases the requirement was for the above sum to be $\leq 0.60 \%$ (21)(22). Cements PY-4 and PY-6 could be so classified (see Table 1).

2. The natural and artificial pozzolans, regardless of their origin and hydraulic factor contents, were chosen to form a $\mathrm{Na}$ and $\mathrm{K}^{\mathrm{i}}$ content gradient, expressed in terms of $[\mathrm{Na}, \mathrm{O}(\%)+(0.658 \times \mathrm{K}, \mathrm{O}(\%))]$, subject to the following additional conditions:

- there was to be a substantial difference between the highest and lowest content, and

- some of the pozzolans were to fail to meet, to a greater or lesser extent, the requirements regarding the maximum allowable alkaline cation content as established in: . the ASINEL proposal (16)(idem $\leq 2.0 \%$ ), or

- ASTM standard C 618-94 a (20) (Na+ and $\mathrm{K}^{*}$ content expressed as $\left.\left[\mathrm{Na}_{2} \mathrm{O}(\%)+\left(0.658 x \mathrm{~K}_{2} \mathrm{O}(\%)\right)\right] \leq 1.50 \%\right)$.

The Frattini test (14) was used to find the pozzolanic: activity index for all the above cements (Table 3), even though this procedure is specified for pozzolanic or CEM-IV cements only (13). Setting times (13) were also found for some of them. The volume of the liquid phase for the Frattini test for the cements blended with pozzolans $D$ and $N$ was substantially smaller than for the rest of the pozzolans tested and/or any traditional pozzolanic cement. Therefore, smaller aliquot parts of the liquid phase than specified for the test had to be used to determine the results for these blended cements. The explanation would have to be sought in the special morphology of these diatomaceous minerals but particularly in their peculiar chemical composition, consisting almost entirely of amorphous silica. 
Adicionalmente se determinaron también los contenidos de $\mathrm{Na}$ y K', en la fase líquida del ensayo de Frattini(14), de las familias de cementos de mezcla constituidos por los cementos Portland P-1,P-2,P-31,PY-4 y PY-6. y las puzolanas $\mathrm{C}$ o $\mathrm{M}$ cuyos contenidos originales de $\mathrm{Na}^{\prime}$ y $\mathrm{K}^{*}$, eran muy dispares. A ellas se les sumó, también con posterioridad, la puzolana $\mathrm{O}$, con el tin de reconfirmar la hipótesis de trabajo, dado que la misma poseía su contenido $\mathrm{N}_{2} \mathrm{O}$ eq. $(\%)$ a continuación que la $\mathrm{C}$ que era la que mayor lo tenía de todas. Los resultados se expresaron en $\mathrm{g} / \mathrm{l}$ y se muestran en la Tabla 4.

Por último, las puzolanas naturales $\mathrm{O}, \mathrm{A}$ y $\mathrm{C}$ tuvieron que ser analizadas por DRX, para ver si su comportamiento en el ensayo de Frattini y en el de los tiempos de fraguado, debido al referido intercambio iónico de iones $\mathrm{Na}^{\prime}$ y $\mathrm{K}$ por $\mathrm{Ca}^{2+}$ o capacidad de cambio, se pudo ver coadyuvado o no por su composición mineralógica, dado que algunas zeolitas. presentes en ciertas puzolanas naturales, se comportan también como puzolanas.

\section{RESULTADOS Y DISCUSIÓN}

Los resultados experimentales obtenidos se encuentran en las Tablas 3 y 4, y su análisis, estudio, discusión e interpretación se va a realizar centrándose especialmente en aquellos casos más apropiados para el objetivo de este trabajo.
In addition, $N a$ and $K^{\prime}$ contents were determined in the Frattini test liquid phase (14) for the P-1, P-2, $P-31, P Y-4$ and PY-6 families of Portland cements blended with the $C$ or $M$ pozzolans with very divergent original $\mathrm{Na}^{-}$and $\mathrm{K}^{-}$contents. The results are given in Table 4.

Finally, X-ray diffraction was used to analyse natural pozzolans $O, A$ and $C$ to ascertain whether their Frattini test and setting time results, attributable to the ion exchange between $\mathrm{Na}^{-} / \mathrm{K}^{\prime}$ and $\mathrm{Ca}$, were reinforced or otherwise by their mineral composition, since some of the zeolites present in certain natural pozzolans also act like pozzolans.

\section{RESULTS AND DISCUSSION}

The experimental results obtained are shown in Tables 3 and 4. The analysis, study, discussion and interpretation of these results focus in particular on the cases best suited to the aims of this study, namely:

TABLA 4/TABLE 4

Fase líquida del ensayo de Frattini: $\mathrm{Na}_{2} \mathrm{O}$ y $\mathrm{K}_{2} \mathrm{O}(\mathrm{g} / \mathrm{l})$ de algunos cementos de mezcla preparados con las puzolanas $\mathrm{C}, \mathrm{O}$ y $\mathrm{M}$

Liquid phase of the Frattini test: $\mathrm{Na}_{2} \mathrm{O}$ and $\mathrm{K}_{2} \mathrm{O}(\mathrm{g} / \mathrm{l})$ of some blended cements prepared with C. $O$ and $M$ pozzolans, respectively

\begin{tabular}{|c|c|c|c|c|c|c|c|c|c|c|c|c|c|c|c|c|c|c|c|c|c|c|c|c|c|c|c|c|c|c|c|}
\hline \multirow{3}{*}{$\begin{array}{l}\text { Edad } \\
\text { (dias) } \\
\text { Ago } \\
\text { (denvs) }\end{array}$} & \multirow{3}{*}{ 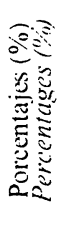 } & \multicolumn{30}{|c|}{ Cementos de Mezcla/Blended Cements } \\
\hline & & \multicolumn{2}{|c|}{$P-1 C$} & \multicolumn{2}{|c|}{$P-10$} & \multicolumn{2}{|c|}{ P-1M } & \multicolumn{2}{|c|}{$P-2 C$} & \multicolumn{2}{|c|}{ P-20 } & \multicolumn{2}{|c|}{ P-2AM } & \multicolumn{2}{|c|}{$\mathrm{P}-31 \mathrm{C}$} & \multicolumn{2}{|c|}{$\mathrm{P}-310$} & \multicolumn{2}{|c|}{ P-31M } & \multicolumn{2}{|c|}{ PY $-4 C$} & \multicolumn{2}{|c|}{ PY- 40} & \multicolumn{2}{|c|}{ PY-4M } & \multicolumn{2}{|c|}{ PY-6C } & \multicolumn{2}{|c|}{ PY-60 } & \multicolumn{2}{|c|}{ PY-6A } \\
\hline & & $\begin{array}{c}\mathrm{Na}_{2} \mathrm{O} \\
(\mathrm{g} g\end{array}$ & $\begin{array}{l}\mathrm{K}_{2} \mathrm{O} \\
\text { (g) }\end{array}$ & $\begin{array}{l}\mathrm{Na}_{2} \mathrm{O} \\
(\mathrm{g} l)\end{array}$ & $\begin{array}{l}\mathrm{K}_{2} \mathrm{O} \\
(\mathrm{g})\end{array}$ & $\begin{array}{l}\mathrm{Na}_{2} \mathrm{O} \\
(\mathrm{g} 1)\end{array}$ & $\begin{array}{l}\mathrm{k}_{2} \mathrm{O} \\
(g)\end{array}$ & $\begin{array}{l}\mathrm{Na}_{2} \mathrm{O} \\
(g)^{\prime}\end{array}$ & $\begin{array}{l}\mathrm{K}_{2} \mathrm{O} \\
(\mathrm{g} \mid)\end{array}$ & $\begin{array}{l}\mathrm{N}_{2} \mathrm{O} \\
(\mathrm{g} l 1)\end{array}$ & $\begin{array}{l}\mathrm{K}_{2} \mathrm{O} \\
(\mathrm{g})\end{array}$ & $\begin{array}{l}\mathrm{Na}_{2} \mathrm{O} \\
\text { (gi) }\end{array}$ & $\begin{array}{l}\mathrm{K}_{2} \mathrm{O} \\
\text { (gl) }\end{array}$ & $\begin{array}{l}\mathrm{Na}_{2} \mathrm{O} \\
(g h)\end{array}$ & $\begin{array}{l}\mathrm{K}_{2} \mathrm{O} \\
(\mathrm{g})\end{array}$ & $\begin{array}{l}\mathrm{N}_{2} \mathrm{O} \\
(\mathrm{g} l)\end{array}$ & $\begin{array}{l}\mathrm{K}_{2} \mathrm{O} \\
\mathrm{ig}(\mathrm{l})\end{array}$ & $\begin{array}{l}\mathrm{Na}_{2} \\
(\mathrm{~g} g\end{array}$ & $\left\{\begin{array}{l}\mathrm{K}_{2} \mathrm{O} \\
(\mathrm{g})\end{array}\right.$ & $\begin{array}{l}\mathrm{Na}_{2} \mathrm{O} \\
(\mathrm{g})\end{array}$ & $\begin{array}{l}\mathrm{K}_{2} \mathrm{O} \\
(\mathrm{g})\end{array}$ & $\begin{array}{c}\mathrm{Na}_{2} \mathrm{O} \\
(\mathrm{g}(1)\end{array}$ & $\begin{array}{l}\mathrm{K}_{2} \mathrm{O} \\
\text { (g) }\end{array}$ & $\left|\begin{array}{c}\mathrm{Na}_{2} \mathrm{O} \\
(\mathrm{g})\end{array}\right|$ & $\begin{array}{l}\mathrm{K}_{2} \mathrm{O} \\
(\mathrm{g})\end{array}$ & $\begin{array}{c}\mathrm{Na}_{2} \mathrm{O} \\
(\mathrm{g})\end{array}$ & $\begin{array}{l}\mathrm{K}_{2} \mathrm{O} \\
(\mathrm{g} 1)\end{array}$ & $\begin{array}{c}\mathrm{Na}_{2} \mathrm{O} \\
(\mathrm{g} h)\end{array}$ & $\begin{array}{l}\mathrm{K}_{2} \mathrm{O} \\
(\mathrm{g}) 1\end{array}$ & $\begin{array}{l}\mathrm{Na}_{2} \mathrm{~d} \\
(\mathrm{~g} g\end{array}$ & $\mid \begin{array}{l}\mathrm{K}_{2} \mathrm{O} \\
(\mathrm{g} 11)\end{array}$ \\
\hline 7 & \multirow{2}{*}{$80 / 20$} & 11,6 & 13,2 & 12,2 & 6,8 & 5,6 & 5,0 & 11,2 & 10,8 & 11,6 & 5,9 & 1,2 & 0,8 & 11,6 & 12,2 & 12,4 & 5,3 & 1,0 & 1,2 & 9,8 & 10,0 & 11,1 & 3,5 & 0,0 & 0,0 & 9,8 & 9,8 & 10,8 & 3,0 & 0,2 & 0,6 \\
\hline 28 & & 14,4 & 16,0 & 15,3 & 8,6 & 6,3 & 14,4 & 14,2 & 14,8 & 14,9 & 8,0 & 1,4 & 7,2 & 15,6 & 20,0 & 16,6 & 9,0 & 1,6 & 14,6 & 13,8 & 10,0 & 15,8 & 3,7 & 0,2 & 2,8 & 13,8 & 9,8 & 15,0 & 3,3 & 3,0 & 9,2 \\
\hline 7 & \multirow{2}{*}{$70 / 30$} & 16,0 & 15,4 & 17,4 & 6,4 & 3,2 & 2,6 & 11,6 & 11,4 & 12,4 & 5,2 & 0,8 & 0,4 & 13,6 & 13,2 & 15,0 & 5,5 & 3,8 & 3,2 & 11,2 & 11,0 & 13,3 & 3,7 & 0,2 & 0,0 & 11,2 & 12,0 & 12,6 & 3,9 & 0,3 & 0,8 \\
\hline 28 & & 17,6 & 14,6 & 19,9 & 7,4 & 4,4 & 12,0 & 110,0 & 15,8 & 12,8 & 7,5 & 0,4 & 4,8 & 14,2 & 13,6 & 15,5 & 5,8 & 3,8 & \begin{tabular}{l|l}
31,6 \\
\end{tabular} & 13,4 & 7,6 & 15,5 & 2,5 & 0,0 & 2,2 & 13,8 & 8,2 & 15,5 & 2,7 & 0,2 & 2,8 \\
\hline 7 & \multirow{2}{*}{$60 / 40$} & 14,8 & 14,8 & 25,5 & 28,5 & 1,2 & 0,8 & 10,0 & 10,2 & 16,0 & 4,4 & 0,4 & 0,2 & 12,6 & 12,8 & 22,0 & 5,0 & 2,8 & 2,4 & 11,2 & 12,2 & 23,4 & 4,6 & 0.6 & 0.4 & 11,8 & 12,2 & 22,5 & 4,4 & 0,6 & 1,0 \\
\hline 28 & & 16,2 & 8,8 & 6,6 & 3,5 & 2,4 & 7,8 & 10,4 & 3,0 & 16,8 & 1,2 & 0,6 & 4,4 & 14,8 & 10,8 & 26,0 & 4,4 & 3,0 & 9,2 & 15,6 & 3,6 & 32,5 & 1,1 & 0,4 & 3,0 & 13,4 & 6,8 & 25,5 & 2,2 & 0,0 & 2,6 \\
\hline
\end{tabular}


1. Las puzolanas $\mathrm{C}$ y $\mathrm{M}$. por este orden, de mayor a menor valor. han fijado, a igualdad de todo lo demás, iones $\mathrm{Ca}^{2}$ en cantidades notablemente superiores a los fijados por el resto de las puzolanas ensayadas. Ambas puzolanas $\mathrm{C}$ y $\mathrm{M}$ son las que posecn los contenidos mayores y menores, respectivamente, de $\mathrm{Na}^{\prime}$ y $\mathrm{K}^{\ddagger}$. Así, los notables contenidos de tales elementos alcalinos de la puzolana $\mathrm{C}$ habrían pasado durante la hidratación de su cemento de mezcla, a la fase líquida del ensayo de Frattini, alcalinizándola y aumentándole su pH, con lo que el producto de solubilidad de la portlandita se alcanzaría, por efecto del ión común, para menores concentraciones de ión $\mathrm{Ca}^{2 \cdot-}$ (la solubilidad del $\mathrm{Ca}(\mathrm{OH})_{2}$, en agua pura es $\left.1.23 \mathrm{~g} / \mathrm{l}\right)$. Por este motivo, la puzolana $\mathrm{C}$ en el ensayo de Frattini, no podrá fijar $\mathrm{Ca}(\mathrm{OH})_{2}$ de una disolución acuosa saturada de $\mathrm{Ca}(\mathrm{OH})_{2}$ puro o muy próxima a la saturación -por tratarse de portlandita y parte disuelta del resto de los componentes mineralógicos del cemento Portland y/o de la propia puzolana-, sino de una disolución saturada de portlandita pero con un menor contenido del catión $\mathrm{Ca}^{2}$. Así y aunque la puzolana $\mathrm{C}$ ha dejado menor cantidad de iones $\mathrm{Ca}^{2}$ libres en la disolución que la $\mathrm{M}$, ello no indica necesariamente que haya tenido mayor capacidad de fijación de tales iones $\mathrm{Ca}^{2}{ }^{2}$ que la $\mathrm{M}$. Ya que la cantidad de iones $\mathrm{Ca}^{2}$ en la disolución depende de la $\left[\mathrm{OH}^{-}\right]$y de la fuerza iónica de la misma, y no sólo, de la capacidad de fijación de las puzolanas.

Por todo ello, la puzolana $\mathrm{C}$ ha demostrado tener una mayor capacidad de cambio de $\mathrm{Na}^{4}$ y $\mathrm{K}^{4}$ por $\mathrm{Ca}^{2+}$ que todas las demás. De aquí que, y según las series liotropas, en la fase líquida de los ensayos de Frattini de la mayor parte de sus cementos de mezcla $\mathrm{PY}-6 / \mathrm{C}, \mathrm{PY}-4 / \mathrm{C}, \mathrm{P}-31 / \mathrm{C}$. P-2/C y P-1/C, la $\left[\mathrm{Na}_{2} \mathrm{O}\right]$ debería de haber aumentado con el transcurso del ensayo y la presencia de puzolana $\mathrm{C}$, mientras que la $\left[\mathrm{K}_{2} \mathrm{O}\right]$ debería haber disminuido; en cambio, en el caso de la puzolana $\mathrm{M}$, no, sino todo lo contrario. $\mathrm{Y}$ eso mismo ha sido lo que ha ocurrido en esta investigación, véase, al efecto, la Tabla 4.

Por otra parte, dicha disminución del ión $\mathrm{K}$ durante el transcurso del ensayo de Frattini, en la fase líquida de la mayor parte de los cementos de mezcla con la puzolana $C$ analizados, deberá llevar aparejado también un aumento del mismo en la correspondiente fase sólida, en detrimento del ión $\mathrm{Na}^{\prime}$, según las referidas series liotropas, el cual en cambio ha tenido que aumentar necesariamente como se ha demostrado, en la correspondiente fase líquida(Tabla 4).

De este modo, el presumible exceso de ión $\mathrm{K}^{\dagger}$ en la fase sólida habría impedido o dificultado que su correspondiente fracción de puzolana $\mathrm{C}$ hubiera fijado una mayor cantidad de ión $\mathrm{Ca}^{2+}$ que la que ha fijado en realidad. Mientras que en la correspondiente fase
1. Pozzolans $C$ and $M$-in that order-fixed appreciably larger amounts of $\mathrm{Ca}^{2}$ than the rest of the pozzolans tested, all other factors being equal. These two pozzolans were the ones with the highest and lowest amounts, respectively, of $\mathrm{Na}^{\dagger}$ and $\mathrm{K}^{+}$. Thus during hydration of the respective blended cement, the high alkali content in pozzolan $C$ would have seeped into and alkalinised the Frattini test liquid phase, raising the $p H$. Therefore, due to the common-ion effect, the portlandite solubility product would be generated at lower concentrations of $\mathrm{Ca}^{2+}(\mathrm{Ca} / \mathrm{OH})_{2}$, solubility in pure water is $1.23 \mathrm{~g} / \mathrm{l})$. For this reason, in the Frattini test, pozzolan $C$ is not able to fix $\mathrm{Ca}(\mathrm{OH})_{2}$, from a saturated or nearly saturated aqueous solution of pure $\mathrm{Ca}(\mathrm{OH})$, or one very close to saturation -since this is portlandite and a dissolved fraction of the remains of mineralogic components of the Portland cement and/or of the pozzolan itself-, but instead a saturated portlandite solution but with a lower $\mathrm{Ca}^{2+}$ cation content. In other words, the fact that pozzolan Cleaves few'er $\mathrm{Ca}^{2}$ ions in solution than $\mathrm{M}$ does not necessarily. mean that it has a greater capacity to fix $\mathrm{Ca}^{2 *}$ ions than $M$. This is because the amount of $\mathrm{Ca}^{2 *}$ ions in solution depends not only on the fixation capacity of the pozzolans, but on the $[\mathrm{OH}]$ concentration and ionic strength as well.

For all these reasons, $C$ was found to have a greater $\mathrm{Na} / \mathrm{K}^{*}$ and $\mathrm{Ca}^{2}$ exchange capacity than all the other pozzolans. Consequently, according to lyotropic (or Hofmeister) series rationale, in the presence of pozzolan $\mathrm{C}$, the $\left[\mathrm{Na}_{2} \mathrm{O}\right]$ in the liquid phase of the Frattini test should rise for most of the blended cements in the series, i.e., $P Y-6 / C, P Y-4 / C, P-31 / C, P-2 / C$ and $P-1 / C$ as the experiment progresses, while $\left[K_{2} \mathrm{O}\right]$ should decline; and vice-versa in the presence of pozzolan $M$. The results of these experiments concurred, in fact, with such expectations (see Table 4).

Moreover; the decline in $K^{\prime}$ concentration in the Frattini test of the liquid phase of most of the cements blended with pozzolan $C$ analysed, should go hand-inhand with an increase in such concentration in the solid phase, to the detriment of ion $\mathrm{Na}^{t}$ which, again according to lyotropic series reasoning, would necessarily have to increase in the respective liquid phase. As Table 4 shows, these expected results were also confirmed.

The presumed excess of $K^{\prime}$ in the solid phase would hav'e prevented or hindered its respective pozzolan C fraction from fixing more $\mathrm{Ca}^{2+}$ than it actually fixed. In the liquid phase, in turn, the higher $\mathrm{Na}$ content should have prevented or hindered $\mathrm{Ca}^{2+}$ concentration 
liquida. la mayor presencia en cambio de ión $\mathrm{Na}$ ', ha debido impedir o dificultar también una mayor presencia de ión $\mathrm{Ca}^{2}$ que la que debería haber habido realmente. Por este motivo, se puede decir con fundamento que el comportamiento de la puzolana $\mathrm{C}$ en el ensayo de Frattini ha sido "más ficticio que real".

Y todo cllo deberá ser tanto más así, cuanto menor sea la causa o causas que determinaron la presencia de ión $\mathrm{Ca}^{2}$ " en dicha fase líquida. entre las cuales cabe citar,

(a) la actividad puzolánica de la propia puzolana,

(b) la cantidad de cemento Portland P o PY, dentro de una misma familia,

(c) la superficie específica Blaine(SEB) del cemento Pórtland, y/o

(d) el contenido, a igualdad de cemento de mezcla, de $\mathrm{C}_{2} \mathrm{~S}$, standard de $\mathrm{Cal}$ y $\mathrm{CaO}_{\text {comll }} ; \mathrm{y}$, a tal efecto, el contenido de $\mathrm{C}_{3} \mathrm{~S}$ del cemento Portland PY-4(58.19\%) es bastante menor que el del PY-6 $(79,43 \%)$, ocurriendo lo contrario, lógicamente, con los contenidos correspondientes de $\mathrm{C}_{2} \mathrm{~S}(19,46 \%>2,29 \%)$, de ambos.

$\mathrm{Y}$, en cuanto al hecho común acaecido a los contenidos de $\mathrm{K}^{\prime}$, tanto

- entre las familias de cementos de mezcla PY-4/C y PY-6/C, como

- entre las familias $\mathrm{P}-1 / \mathrm{C}$ y $\mathrm{P}-31 / \mathrm{C}$ respecto de la $\mathrm{P}-2 / \mathrm{C}$.

se pudo ver que resultó ser una confirmación más de que la causa residió en ambas cuatro anteriores.

Habiendo sido, no obstante, la más evidente de todas la que se refiere al contenido de $\mathrm{C}_{3} \mathrm{~S}$-menor de $\mathrm{C}_{3} \mathrm{~S}$ y mayor de $\mathrm{C}_{2} \mathrm{~S}$ - de ambos cementos Portland matrices PY-4 y P-2, respecto de los demás correspondientes PY-6, P-1 y P-31, respectivamente, con cada uno de ellos comparados, además de la menor SEB del P-2 respecto del P-1 y del P-31, principalmente, lo cual va a favor de la razón aducida.

Y para poder verificar la hipótesis de comportamiento expuesta, debería haberse producido el hecho de que a las correspondientes fases líquidas de los cementos de mezcla de la puzolana $\mathrm{O}$ (que era la puzolana que poseía menor contenido de $\mathrm{Na}_{2} \mathrm{O}$ eq. $(\%)$ que la $\mathrm{C} \mathrm{y}$ mayor que el resto(Tabla 5), siendo además menos activa que la $\mathrm{C}$ ), debería haberles ocurrido otro tanto, sólo que corregido y disminuido en valores absolutos y número de casos, como asi ha ocurrido en esta investigación, véase, al efecto, la Tabla 4.

Todo lo cual se debe traducir por tanto en relación al parámetro "ticmpos de fraguado", que este otro parámetro deberá ser más proclive a la hipótesis de comportamiento expuesta, cuando las puzolanas $\mathrm{C}$ y $\mathrm{O}$ especialmente, se mezclen con un cemento Portland from rising higher than it actually should have. For this reason, it can be sustained that the hehaviour of pozzolan $C$ in the Frattini test was "more apparent than real".

This effect should be greater when the cause or causes determining the presence of $\mathrm{Ca}^{2+}$ in the liquid phase are less intense. These causes include:

(a) the pozzolanic activity of pozzolan $C$ itself;,

(b) the amount of Portland cement $P$ or $P Y$, in a given family

(c) the Blaine Specific Surface (BSS) of the PC, and/or (d) the content, for the same blend of cement, of $C_{3} S$, standard lime and $\mathrm{CaO}_{\text {comb. }}$; in this regard, the $\mathrm{C}_{3} \mathrm{~S}$ content of Portland cement PY-4 (58.19\%) is considerably lower than in PY-6 (79.43\%) and viceversa, logically, for their respective $C_{2} S(19.46 \%>2.29 \%)$ contents.

And the common findings observed with respect to $K^{4}$ concentration for both

\section{- blended cement families $P Y-4 / C$ and $P Y-6 / C$, and \\ - families $P-1 / C$ and $P-31 / C$ compared to $P-2 / C$,}

provided further confirmation of the importance of the above four factors. The clearest finding in this regard. in any case, is the $C_{3} S$ content -the larger the amount of $C_{3} S$ the smaller the amount of $C_{2} S$ - in the two Portland cements $P Y-4$ and $P-2$, with respect to $C_{3} S$ content in $P Y-6, P-1$ and $P-31$, compared one by one. The smaller $B S S$ in $O P C P-2$ compared to $P-1$ and $P-31$ corroborates this reasoning.

Further verification of the above hypothesis can be sought in the results found for the liquid phases of the cements blended with pozzolan $O$ (the pozzolan with lower $\mathrm{Na}_{2} \mathrm{O}$ eq. $(\%)$ content than $\mathrm{C}$ but higher than all the rest -Table 5-, which should have been similar to the findings for pozzolan $C$ blended cement, but with smaller absolute values and number of cases due to the fact that pozzolan $O$ was less active than pozzolan $C$. And this, indeed, was observed in the experimental results (see Table 4).

All of the above should translate into "setting times" that reflect the hypothesis described above more closely when pozzolans $C$, and especially $O$, are mixed with ordinary Portland cement (OPC) such as P-I, than when they are mixed with sulfate-resistant cement 
ordinario (CPO), caso por ejemplo del P-1, que cuando se mezclen con un cemento resistente a los sulfatos (CPRS), caso por ejemplo del PY-6, como así ha ocurrido también en csta investigación, véase, al efecto, la Tabla 6.

Por último y si a todo ello se le añade la muy importante causa circunstancial adicional de que mediante la técnica analítica de DRX, se pudo demostrar que uno de los componentes cristalinos significativos de la puzolana $\mathrm{C}$ cra una zeolita $\left[\mathrm{N}_{2} \mathrm{O}-\mathrm{Al}_{2} \mathrm{O}_{3}-\mathrm{SiO}_{2}-\mathrm{H}_{2} \mathrm{O}\right]$ además de la phillipsita $\left[\mathrm{K}_{2}^{2} \mathrm{Ca}_{2}(\mathrm{Al}, \mathrm{Si})_{16} \mathrm{O}_{32} \cdot 13,5 \mathrm{H}_{2} \mathrm{O}\right]$, queda pues justificada más aún, la posibilidad de existencia de capacidad de
(SRC) such as PY-6. And again, the experimental results corroborate this expectation, as can be seen in Table 6.

Finally, the highly relevant circumstantial fact that $X R D$ proved that one of the significant crystalline components of pozzolan $C$ was a zeolite $\left[\mathrm{N}_{2} \mathrm{O}-\mathrm{Al}_{2} \mathrm{O}_{3}-\mathrm{SiO}_{2}-\mathrm{H}_{2} \mathrm{O}\right]$ and that phillipsite $\left[\mathrm{K}_{2} \mathrm{Ca}_{2}(\mathrm{Al}, \mathrm{Si}){ }_{16} \mathrm{O}_{32} .13,5 \mathrm{H}_{2} \mathrm{O}\right]$ was likewise present constitutes further substantiation of the possibility that some pozzolans may have exchange capacity. The

TABLA 5/TABLE 5

Ensayo de Frattini: clasificación de las puzolanas D, N, O, A, C y $\mathrm{M}$ en función de diferentes parámetros químicos

Frattini test: classification of $D, N, O, A, C$ and $M$ pozzolans in relation to different chemical parameters

\begin{tabular}{|c|c|c|c|c|}
\hline \multirow{3}{*}{ 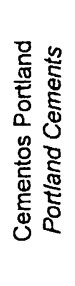 } & \multirow{3}{*}{ 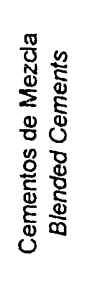 } & \multicolumn{2}{|c|}{ Ensayo de Frattini/Frattini test } & \multirow{3}{*}{$\mathrm{N}^{c}$} \\
\hline & & \multicolumn{2}{|c|}{$\begin{array}{l}\text { Clasificación de las Puzolanas } D, N, O, A, C \text { y } M \\
\text { en función del contenido de: } \\
\text { Classification of } D, N, O, A, C \text { and } M \text { pozzolans } \\
\text { in terms of: }\end{array}$} & \\
\hline & & {$[\mathrm{CaO}](\mathrm{mM} / \mathrm{l})$ content } & [OF ] $(\mathrm{mM} / \mathrm{l})$ content & \\
\hline \multirow{3}{*}{$\mathrm{P} 1$} & $80 / 20$ & $C<A<N<M<O<D$ & $N<A<M<C<D<O$ & 1 \\
\hline & $70 / 30$ & $M<C<N<A<0<D$ & $N<M<D<A<C<O$ & 2 \\
\hline & $60 / 40$ & $M<N<C<O<A<D$ & $N<M<D<A<O<C$ & 3 \\
\hline \multirow{3}{*}{ P2 } & $80 / 20$ & $C<M<N<D<A<O$ & $N<M<D<A<C<O$ & 4 \\
\hline & $70 / 30$ & $C<M<N<D<A<0$ & $N<M<D<A<O<C$ & 5 \\
\hline & $60 / 40$ & $C<M<N<D<A<O$ & $N<A<D<M<O<C$ & 6 \\
\hline \multirow{3}{*}{ P31 } & $80 / 20$ & $C<N<D<M<O<A$ & $N<D<A<M<O<C$ & 7 \\
\hline & $70 / 30$ & $C V-10<C<C V-19<M<D<N<A<O$ & $N<C V-19<C V-10<M<D<A<O<C$ & 8 \\
\hline & $60 / 40$ & $C<M<D<N<A<O$ & $N<M<A<D<O<C$ & 9 \\
\hline \multirow{3}{*}{ PY4 } & $80 / 20$ & $C<M<A<O<N<D$ & $N<M<O<D<C<A$ & 10 \\
\hline & $70 / 30$ & $C<M<O<A<N<D$ & $\mathrm{~N}<\mathrm{M}<\mathrm{A}<\mathrm{D}<\mathrm{O}<\mathrm{C}$ & 11 \\
\hline & $60 / 40$ & $C<M<O<A<N<D$ & $\mathrm{~N}<\mathrm{M}<\mathrm{O}<\mathrm{A}<\mathrm{D}<\mathrm{C}$ & 12 \\
\hline \multirow{3}{*}{ PY6 } & $80 / 20$ & $C<N<M<D<O<A$ & $N<M<D<A<O<C$ & 13 \\
\hline & $70 / 30$ & $C<M<N<O<D<A$ & $N<M<D<A<O<C$ & 14 \\
\hline & $60 / 40$ & $C<M<N=O<D<A$ & $\mathrm{~N}<\mathrm{M}<\mathrm{D}<\mathrm{A}<\mathrm{O}<\mathrm{C}$ & 15 \\
\hline \multicolumn{2}{|c|}{$\begin{array}{l}\text { Contenido de } \mathrm{Na}_{2} \mathrm{O} \text { ec } \\
\text { de la Puzolana } \\
\mathrm{Na}_{2} \mathrm{O} \text { eq. (\%) content } \\
\text { of the Pozzolan }\end{array}$} & \multicolumn{2}{|c|}{$N=M<A<D<O<C$} & 16 \\
\hline
\end{tabular}


cambio en algunas puzolanas. $Y$ decimos circunstancial, porque las puzolanas $\mathrm{O}$ y $\mathrm{A}$ no las poseían, y no por ello dejaron de estar cerca de la $\mathrm{C}$, en la mayor parte de las diferentes clasificaciones obtenidas, véanse, al efecto, las Tablas 5 y 6 .

2. No obstante lo anterior, en el estudio comparativo similar realizado a las puzolanas $\mathrm{O}$ y $\mathrm{A}$, se ha podido observar otro tanto, aunque en menor medida. Pero en este caso a favor de la $\mathrm{O}$, que, al haber originado mayores valores de alcalinidad $\left[\mathrm{OH}^{-}\right]$, que la $\mathrm{A}$ (Tabla 5), confirmó su mayor semejanza con la $\mathrm{C}$ que la $\mathrm{A}, \mathrm{y}$, por consiguiente, habrá podido participar más que la $\mathrm{A}$, de alguna(s) de las causas tan sólo del circumstantial nature of this finding must be stressed here, since neither pozzolan $O$ nor $A$ contained these minerals, and nonetheless had values close to the values recorded for $C$ in most of the classifications (see Tables 5 and 6).

2. Notwithstanding the foregoing, the comparative study of pozzolans $O$ and $A$ revealed similar qualitative although quantitatively mitigated results. In this case, pozzolan $\mathrm{O}$, which generated higher alkalinity [OH $\mathrm{OH}^{-}$, proved to be closer in behaviour to $C$ than to A (Table 5) and therefore must have shared, more than $A$, in some but not all of the properties of $C$ that

\section{TABLA 6/TABLE 6}

Clasificaciones de las puzolanas ensayadas en función de los tiempos de fraguado de algunos de los cementos de mezcla con el CP P-1

Classifications of the pozzolans tested in terms of setting times for some blended cements with common PC P-1

\begin{tabular}{|c|c|c|c|}
\hline 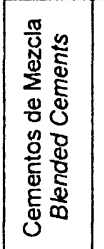 & 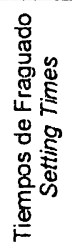 & $\begin{array}{l}\text { Clasificaciones } \\
\text { Classifications }\end{array}$ & $\mathrm{N}^{\circ}$ \\
\hline $\begin{array}{l}P-1 / Z^{n} \\
80 / 20\end{array}$ & \multirow{2}{*}{ 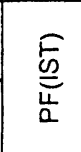 } & $D^{*}>0>C>M=C V-10>C V-19>A$ & 1 \\
\hline \begin{tabular}{r|}
$-1 / Z^{n}$ \\
$60 / 40$
\end{tabular} & & $D^{*}>M=C V-19>C V-10>0>C>A$ & 2 \\
\hline $\begin{array}{r}P-1 / Z^{n} \\
80 / 20\end{array}$ & \multirow{2}{*}{$\begin{array}{c}\tilde{5} \\
\frac{w}{4} \\
\frac{u}{4}\end{array}$} & $D^{*}>C V-19>M=C V-10>0>A>C$ & 3 \\
\hline $\begin{array}{c}P-1 / Z^{n} \\
60 / 40\end{array}$ & & $D>A>M=C V-19>C V-10>0>C$ & 4 \\
\hline $\begin{array}{r}P-1 / Z^{n} \\
80 / 20\end{array}$ & \multirow{2}{*}{$\begin{array}{c}\frac{E}{E} \\
\stackrel{L}{L}\end{array}$} & $C V-19>D{ }^{*}>M=C V-10=A>O=C$ & 5 \\
\hline $\begin{array}{c}P-1 / Z^{n} \\
60 / 40\end{array}$ & & $D>A>M=C V-19>0>C V-10>C$ & 6 \\
\hline $\mid \begin{array}{r}P Y-6 / " Z \\
80 / 20\end{array}$ & \multirow{2}{*}{ 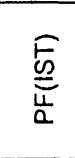 } & $D>A>C \stackrel{*}{>} M>0>C V-10>C V-19$ & 7 \\
\hline \begin{tabular}{|r|}
$\mathrm{PY}-6 / \mathrm{Z}$ \\
$60 / 40$ \\
\end{tabular} & & $D>M>C V-10>C>0>C V-19>A$ & 8 \\
\hline $\begin{array}{c}\text { PY-6/ZZ' } \\
80 / 20\end{array}$ & \multirow{2}{*}{$\begin{array}{l}\text { E } \\
\frac{4}{4} \\
\frac{4}{4}\end{array}$} & $D>M>C V-10>C V-19>C>A>O^{*}$ & 9 \\
\hline $\begin{array}{c}\mathrm{PY}-6 / \mathrm{Z} \\
60 / 40\end{array}$ & & $D>M>C V-10>C V-19>C>0>A$ & 10 \\
\hline $\begin{array}{r}\text { PY-6/"Z } \\
80 / 20 \\
\end{array}$ & \multirow{2}{*}{$\frac{\vec{E}}{\frac{E}{L}}$} & $C V-10>C V-19>M>O>D>C>A$ & 11 \\
\hline $\begin{array}{r}P Y-6 / 2 \\
60 / 40\end{array}$ & & $C V-19>A>D>0=C V-10>M>C^{*}$ & 12 \\
\hline \multicolumn{4}{|c|}{ *.- Cemento portland P-1 ó PY-6 (Plain OPC P-1 or SRPC PY-6) } \\
\hline
\end{tabular}


comportamiento de la $\mathrm{C}$ en este ensayo de Frattini, mencionadas antes. Porque la puzolana $O$ no poseía zeolita alguna en su constitución mineralógica, ni la $\mathrm{A}$ tampoco, mientras que la $\mathrm{C}$ sí, como se acaba de ver. Además, a tal efecto. se debe de tener en cuenta también la mayor proximidad al mar Mediterráneo de las canteras de la puzolana O (de Olot-Gerona) que las de la A (de Almagro-Ciudad Real). mientras que el origen de la $\mathrm{C}$ es las Islas Canarias que, como es sabido, se encuentran en cl Océano Atlántico.

Y para mayor abundamiento, véase. en la Tabla 5, cómo las diferentes clasificaciones que se han obtenido, a igualdad de edad y cemento de mezcla, de las puzolanas D, O, A, C y M, en función del valor de la alcalinidad. $\left[\mathrm{OH}^{-}\right]$, de su fase líquida respectiva, han sido casi todas coincidentes con la que se obtendría en función de su contenido de $[\mathrm{Na}, \mathrm{O}(\%)+(0,658 \times \mathrm{K}, \mathrm{O}(\%))]$. De modo y manera que, según todo lo anterior, es lógico que el extremo de mayor valor de alcalinidad haya sido ocupado casi siempre por la $\mathrm{C}$. (en 11 clasificaciones, de un total de 15). seguida de la $\mathrm{O}$ y la $\mathrm{A}$ en dicho orden y número de veces (en 3 clasificaciones y 1, respectivamente). Por este motivo, resultaría lógico también que en el análisis y estudio comparativo de las mismas, realizado en función de los finales y tiempos de fraguado, principalmente, de sus cementos de mezcla con el cemento Portland P-1 en especial (Tabla 6), los de la puzolana $\mathrm{C}$ fueran los menores de todos, seguida a corta o muy corta distancia casi siempre por la $\mathrm{O}$, según el cemento de mezcla que se considere, o la $\mathrm{A}$, si bien ésta en menor número de ocasiones.

A tal efecto.

- en el caso de los 80/20, todos los finales de fraguado ( $F F$ ) estuvieron comprendidos entre 4 horas 20 minutos (4 horas 20 minutos) y 5 horas 40 minutos, y los tiempos de fraguado (TF) estuvieron entre 1 hora 20 minutos y 2 horas 10 minutos, mientras que - en el caso de los 60/40, los FF estuvieron entre 3 horas 50 minutos y $>8$ horas, y los TF entre lhora 25 minutos y más de 8 horas.

En cambio, en el caso del comportamiento de sus correspondientes cementos de mezcla frente al ataque de los sulfatos (2) (3) (23) y calor de hidratación (24), en los que sus contenidos de $\mathrm{Na}^{\prime}$ y $\mathrm{K}$ ' priman bastante menos que los de sus principales factores hidráulicos, la clasificación de las puzolanas ensayadas de mejor a peor comportamiento, a igualdad de todo lo demás, fue la siguiente:

$$
\mathrm{D}>\mathrm{N}>\mathrm{C}>\mathrm{C} \mathrm{V}-19>\mathrm{A}>\mathrm{C}>\mathrm{CV}-10>\mathrm{M}
$$

Así y de acuerdo con dicha caracterización sulfática (2) (3) (23), mientras que a la puzolana $O$ se le tuvo que adscribir el calificativo de "silico-aluminosa", para así poderla diferenciar de las "silícicas" D y N, a la C y a la contribute to the Frattini test results, inasmuch as neither $O$ nor $A$ had zeolite in their composition. Moreover; the pozzolan O quarries (in Olot, Gerona, a province on Spain's northeastern coast) are closer to the Mediterranean Sea than the A quarries (at Almagro, Ciudad Real, an interior province), whilst pozzolan $C$ is from the Canary Islands (Atlantic Ocean).

Furthermore, as Table 5 shows, the various classifications of $D, O, A, C$ and $M$ for any given type of cement and paste age, based on the alkalinity, $\left[\mathrm{OH}^{-}\right]$, of their respective liquid phases, nearly always concurred with their $[\mathrm{Na}, \mathrm{O}(\%)+(0.658 \times \mathrm{K}, \mathrm{O}(\%))]$ content. In light of all the foregoing, pozzolin $C$, logically; showed the highest alkalinity in practically: all cases (II out of a total of 15), followed by $O$ and $A$ in that order (highest alkalinity in 3 classifications and I classification, respectively). For these same reasons, the setting times and final setting times for pozzolan $C$ blended Portland cement, P-I in particular; would be cxpected to be shorter than for all the rest, followed very closely by the pozzolan $O$ blends, or $A$, depending on the cement.

In this respect,

- for 80/20 POZC blends, all final setting times were between 4 hours and 20 minutes (4:20) and 5:40, and setting times ranged from $1: 20$ to $2: 10$, where as

- for 60/40 POZC, final setting times ran from 3:50 to > $8: 00$, and setting times from $1: 25$ to $>8: 00$.

By contrast, with respect to the behaviour in response to sulfate attack (2) (3) (23) and heat of hydration (24) in blended cements where the pozzolan $N \mathrm{~N}^{\prime}$ and $\mathrm{K}^{\prime}$ content was considerably less significant than the hydraulic factors of the cement itself, all other conditions being equal, the classification obtained for the pozzolans (2) (3) (23) (24), from best to worst behaviour was:

Pursuant to this characterisation (2) (3) (23), pozzolan O was classified as alumino-siliceous, to differentiate it from the "siliceous" pozzolans I) and $N$ and pozzolans 
CV-10 se les tuvo que adscribir el calificativo de "alumino-silícica". para así poderlas diferenciar de la "alumínica" $\mathrm{M}$, es decir, a ambas puzolanas $\mathrm{O}$ y $\mathrm{C}$ se les tuvieron que adscribir calificativos opuestos dado su opuesto comportamiento frente al ataque de los sulfatos de sus correspondientes cementos de mezcla.

En resumen, como se puede ver, las clasificaciones

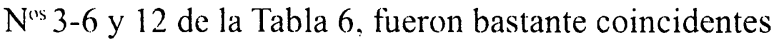
con las clasificaciones $\mathrm{N}^{\mathrm{os}} 3-15$ y 16 de la Tabla 5 , debido a la misma razón química: los notables contenidos de $\mathrm{N}_{2} \mathrm{O}(\%)$ y/o $\mathrm{K}_{2} \mathrm{O}(\%)$ en las puzolanas $\mathrm{O}$ y $\mathrm{C}$, principalmente, y la menor y mayor, respectivamente, capacidad de cambio que promovieron por motivo (s) diverso (s) durante su hidratación con los cementos Portland, y muy en especial con el P-1, tal y como era lo lógico. En cambio no fueron nada coincidentes sino todo lo contrario. con la clasificación anterior [1], tal y como era lo lógico también.

3. Que las puzolanas CV-19 y CV-10, han participado también en igual medida aunque menor cuantía, de las razones dadas para el comportamiento mostrado por las puzolanas $\mathrm{C}$ y $\mathrm{M}$, en la fijación de $\mathrm{Ca}^{2-}$ y $\mathrm{OH}^{-}$.

En definitiva, los resultados aquí obtenidos coincidieron básicamente con los resultados que P. J. Nixon y cols.(25) obtuvieron sobre el efecto de cementos con cenizas volantes con elevados contenidos de alcalinos, sobre la disolución de los poros y sobre la reactividad árido-álcali.

Pero no es menos cierto tampoco, que notables contenidos de alcalinos de una puzolana natural o artificial, lixiviables por un medio básico o simplemente neutro, influyen además, por lo general, negativamente, entre otros parámetros, en el calor de hidratación (24) (26) (27) (28), en las resistencias mecánicas (13) (29) y en la durabilidad (reactividad árido-álcali (25), carbonatación, ataque por cloruros a las armaduras, etc.,etc.) de los cementos y/o de sus productos derivados hormigones y morteros para la construcción, que, con la misma, se preparen.

Únicamente y en principio, los tiempos de fraguado y la protección de las armaduras podrían verse acortados y aumentada, respectivamente, si bien esta última bajo ciertas condiciones, como, por ejemplo, que no se vean acompañados por cloruros de origen marino o no, y que el entorno medioambiental circundante sea seco. Pues en caso contrario, como, por ejemplo, ambiente marino, estudios, investigaciones y experiencias recientes (9) (10) (30), apuntan la necesidad de llevar a cabo una investigación técnica más realista, racional y con una metodología más sistemática, que se fundamente en tales avances científicos, pero, sobre todo, que incluya necesariamente un número mínimo de adiciones puzolánicas o no puzolánicas, que sea representativo de
$C$ and CV-10 were classified as "siliceo-aluminous" to differentiate them from the "aluminous" pozzolan M. In other words, pozzolans $O$ and $C$ were classified differently on the grounds of their different behaviour in response to the sulfate attack in their respective blended cements.

In short, classifications 3-6 and 12 in Table 6 concurred essentially with classifications 3-15 and 16 in Table 5 for the same chemical reason: the high $\mathrm{Na}, \mathrm{O}(\%)$ and/or $\mathrm{K}, \mathrm{O}(\%)$ content in pozzolans $\mathrm{O}$ and especially $C$, and the-respectivelv-lesser and greater exchange capacity generated during their hydration with the PC, and particularly with the OPC P-1, as expected. They were not at all concurrent, however, with the classification shown above as [1], likewise as expected.

\section{Although in quantitatively smaller measure, the} reasons that explain the behaviour of pozzolans $C$ and $M$ with regard to $\mathrm{Ca}^{2+}$ fixation and $\mathrm{OH}^{-}$concentration are likewise valid for pozzolans $C V-19$ and $C V-10$.

The results obtained here essentially concurred with the findings reported by $P$. J. Nixon et al. (25) regarding the effect of fly ash concrete with high alkali content on pore solution and alkali-aggregate reactivity:

It is ronetheless also true that a high alkali content in a natural or artificial pozzolan, which can leach into a basic or even a neutral medium, has an adverse effect on, among others, heat of hydration (24) (26) (27) (28), mechanical strength (13) (29) and cement and/or concrete and mortar durability (ASR (25), carbonation, chloride attack on reinforcing steel, etc.). The benefits, initially, are shorter setting times and greater protection for reinforcement, subject in the latter case to certain conditions, such as the absence of chlorides of marine or any other origin and the existence of a dry surrounding environment. In all other cases, in marine environments, for instance, as recent studies, research and experiments (9)(10)(30) suggest, more realistic, rational and systematic technical research is required, based on the latest scientific methodology, which should cover a large enough number of pozzolanic and 
todas ellas -como se ha hecho para esta investigación y para otras muchas anteriores (2) (3) (8) (23) (24) (31) (32)-, y no sólo para unas pocas. Pues no en vano se trataría de ratificar o precisar más aún, reglamentaciones y especificaciones españolas en vigor (13) (37) (38), que pese a todo, aún se encuentran en fundamentada controversia (30).

\section{CONCLUSIONES}

1. Fl concepto de capacidad de cambio de las arcillas no es íntegramente aplicable a las puzolanas.

Únicamente es aplicable de una manera parcial. Y sólo a aquéllas que posean, por su origen, unos elevados contenidos de alcalinos $\mathrm{Na}$ y/o K en su constitución química que sean lixiviables en medio básico o neutro y/o posean zeolita (s) en su constitución mineralógica.

La razón es que los tratamientos químicos necesarios para su determinación. no le afectan para nada al estado eminentemente cristalino de las arcillas, mientras que al estado eminentemente vítreo y/o amorfo de las puzolanas, si les pueden afectar. Ilasta el punto de hacer que se transformen, parcial o totalmente, en múltiples y variados compuestos cristalinos nuevos.

No obstante y a pesar de lo anterior, en el rango que únicamente le es aplicable a algunas puzolanas el concepto de capacidad de cambio, ćstas participan íntegramente también de su fundamento científico: la capacidad de intercambio cationico que se produce con la correspondiente lase líquida que la circunde, durante su hidratación con cemento Portland. Y cuando esto ocurre, su actividad puzolánica propiamente dicha, se ve menoscabada. Lo que puede afectar a su comportamiento tecnológico: químico, físico y/o de resistencias mecánicas y/o durabilidad (ataque por sulfatos, cloruros, agua de mar, carbonatación, reacción árido-álcali, estabilidad de volumen. tiempos de fraguado, desprendimiento de calor de hidratación, etc., etc.)

2. El contenido de $\mathrm{C}_{3} \mathrm{~A}$ del cemento Portland con el que se mezcló la puzolana de turno -natural o artificial-, no resultó ser determinante para mejor o para peor, en este otro ensayo de Frattini a las edades de 7 y/o 28 días, no pareciendo haber ejercido influencia significativa alguna en la aparición o no de la capacidad de cambio en cuestion a dichas edades.

En cambio, a edades del ensayo menores e incluso muy tempranas, como, por ejemplo, en las primeras 24 ó 48 horas, sí con alguna (s) de ellas, la $\mathrm{O}$ y la $\mathrm{C}$ especialmente, como así lo han confirmado los tiempos de fraguado de sus cementos de mezcla(13) (29), y además. los resultados de las recientes investigaciones non-pozzolanic additions to be representative of all rather than only a few such additions -as in the case of this and previous studies (2) (3) (8) (23) (24) (31) (32). One relevant ultimate aim would be to ratify or adjust certain Spanish regulations and specifications presently in effect (13) (37) (38) in this regard, whose validity has been justifiably challenged (30).

\section{CONCLUSIONS}

1. Exchange capacity, a concept defined for clays, is not wholly applicable to pozzolans. It is, however, partially applicable to those whose high $\mathrm{Na}$ and/or $K$ content leach into basic or neutral media and/or which contain zeolite(s) in their mineral composition.

The reason for this is that whilst the chemical treatments required to determine such capacity do not affect clay crystals, they do impact vitreous and/or amorphous pozzolans, partially or totally converting them into a variety of new crystalline compounds.

Notwithstanding the above, to the sole extent to which the concept is applicable to some pozzolans, the underlying scientific fundamentals are equally valid in these materials: the cationic exchange capacity generated with the respective surrounding liquid phase during hydration with Portland cement. And when this occurs, the pozzolanic activity per se is reduced. And this may affect the performance of the addition, in terms of chemical or physical properties and/or mechanical strength and/or durability (sulfate attack, chlorides, sea water, carbonation, alkali-aggregate. reaction, volume stability, setting times, release of heat of hydration and so on).

\section{The C. A (\%) content in the plain PC with which} each natural or artificial pozzolan was blended had no decisive effect, good or bad, on the Frattini test results at 7 or 28 days, and did not appear to have any significant influence on the presence or otherwise of exchange capacity at such ages.

However at younger and even very early ages, such as 24 or 48 hours after mixing, this parameter did have an effect on some of the pozzolans, such as $O$ and especially $C$, a fact confirmed by the setting times of the respective blended cements (13) (29), and by recent 
realizadas al efecto sobre el calor de hidratación (24) $(26-28)$, ataque por sulfatos (2-7) (33-36) y ataque por cloruros (8) (31).

3. En general, a igualdad de edad del ensayo de Frattini, cemento de mezcla 60/40 y cemento Portland que lo constituya, las puzolanas con las que menores cantidades de $\mathrm{Ca}^{2}$ 'se han determinado en la fase líquida de dicho ensayo, fueron la $\mathrm{C}$ y la $\mathrm{M}$, seguidas, a una relativa distancia. por la puzolana $\mathrm{N}, \mathrm{y}$, a una mayor, por el resto.

4. No obstante la conclusión anterior, la causa por la que la puzolana $C$ ha mostrado un mejor comportamiento que la $\mathrm{M}$ en este ensayo de Frattini, en contra de lo que se esperaba según su comportamiento ante el ataque por separado de sulfatos (2) (3) y cloruros (8) (31) y calor de hidratación (24) (26), que habían sido investigados antes, ha sido su notable mayor contenido de cationes $\mathrm{Na}^{\prime}$ y $\mathrm{K}$, junto con sus contenidos de zcolita y phillipsita. Ambos cationes, al haberse ido "intercambiando" en un cierto orden durante el transcurso del ensayo, según las series liotropas, con cationes $\mathrm{Ca}^{2}$ de la fase líquida del mismo -es decir, ha habido una cierta capacidad de cambio por ambas causas a la vez-, han influido,

(a) elevando notablemente su alcalinidad y $\mathrm{pH}, \mathrm{y}$ paralelamente.

(b) disminuyendo más que la puzolana $\mathrm{M}$, y de un modo también notable, el nivel de saturación de portlandita, $\mathrm{Ca}(\mathrm{OH})_{2}$, de dicha fase líquida,

habiendo concluido, por tanto, que la puzolana $\mathrm{C}$ cumpliera el ensayo de Frattini, paradójicamente, mejor que la $\mathrm{M}$, aunque, de acuerdo con lo anterior, dicha cumplimentación o comportamiento positivo en dicho ensayo se puede decir, en verdad, que ha sido más "aparente o fictico" que real.

5. En el estudio comparativo similar realizado a las puzolanas $\mathrm{O}$ y A, se ha podido observar otro tanto, aunque en apreciable menor medida -a lo ocurrido entre la $\mathrm{C}$ y la $\mathrm{M}-$. Pero, en este otro caso, a favor de la $\mathrm{O}$. Que. al haber originado mayores valores de alcalinidad de la fase líquida que la $\mathrm{A}$, ha confirmado su mayor semejanza -según los parámetros alcalinidad $\left[\mathrm{OH}^{-}\right]$, y tiempos de fraguado de sus cementos de mezcla con el CPO P-1, especialmente, como es lógico- con la $\mathrm{C}$ que con la $\mathrm{A}, \mathrm{y}$, por consiguiente, habrá podido participar más que la A. de alguna (s) de las causas tan sólo, del comportamiento de la $C^{\prime}$ en este ensayo, dadas anteriormente. Porque la puzolana O no poseía zeolita alguna en su constitución mineralógica y la $\mathrm{C}$ si, véase, al efecto. la conclusión 4 .

6. Las puzolanas $\mathrm{CV}-10$ y $\mathrm{CV}-19$ han participado en igual medida, aunque en mucha menor cuantía, de las research on heat of hydration (24) (26) (27) (28) and sulfate (2-7)(33-36) and chloride attack (8)(31).

\section{Generally speaking, for any given Frattini test age} and type of Portland cement, with a mix ratio of 60/40, the pozzolans showing the smallest amounts of $\mathrm{Ca}^{2-*}$ in the test liquid phase were $C$ and $M$, followed, relatively closely, by pozzolan $N$, and at some distance by all the others.

4. Notwithstanding the above conclusion, pozzolan $C$ performed better than $M$ in the Frattini test, despite the expected behaviour in response to the separate attack by sulfates (2)(3) and chlorides (8)(31) and the effect of heat of hydration, which had been studied in previous research, because of its clearly higher $\mathrm{Na}^{\prime}$ and $\mathrm{K}^{+}$ content, together with the presence of zeolite and phillipsite in its mineral composition. The two ions were "exchanged" during the test, in accordance with lyotropic series order, for the $\mathrm{Ca}^{\text {? }}$ cations in the liquid phase -i.e., some exchange capacity was generated for both reasons. As a result these ions contributed to:

(a) a pronounced increase in alkalinity and $p H$, and, at the same time.

(b) a reduction, more than observed in pozzolan $M$, in the portlandite $-\mathrm{Ca}(\mathrm{OH})$, - saturation of this liquid phase.

It may be inferred from the above that $C$ pozzolan performs better under the Frattini test than pozzolan $M$, although as seen from the foregoing discussion, this behaviour would appear to be "more apparent than real".

5. The results of a comparative study of pozzolans $O$ and $A$ were qualitatively similar to the findings for the comparison between $C$ and $M$, although quantitatively more moderate. In this case, pozzolan $O$, which generated higher alkalinity than $A$, proved to be closer in terms of alkalinity, $[\mathrm{OH}]$, and setting times of its respective blended cements, especially -as would be expected- OPC P-1, to $C$ than to $A$. Therefore, $O$ was more closely related than $A$ to $C$ in only some of the properties contributing to these test results, inasmuch as $O$ contained no zeolite in its composition, whereas $C$ in fact did: see conclusion 4.

6. Although in smaller measure, the behaviour of pozzolans $\mathrm{CV}-19$ and $\mathrm{CV}-10$ with regard to liquid 
razones del comportamiento dadas para la $\mathrm{C}$ y $\mathrm{M}$, en la fijación de Ca” de la fase liquida asi como en la alcalinidad de la misma.

7. De acuerdo con los resultados obtenidos del ensayo de Frattini. las ocho puzolanas estudiadas se pueden agrupar, a igualdad de cemento de mezcla. en dos grupos. en función de su actividad puzolánica. entendiéndose por tal. la mayor fijación de iones $\mathrm{Ca}^{2}$ y evolución de la alcaliniad. $\mathrm{OH}^{-}$. Esos dos grupos scrian los siguientes:

(a) En cuanto a la fijación de iones 'a $^{2}$ o evolución de la $|\mathrm{CaO}|$ y la correspondiente evolución de la alcalinidad $\left|\mathrm{OH}^{-}\right|$:

- Grupo formado por las puzolanas $C \mathrm{~V}-10 . \mathrm{CV}-19 . \mathrm{N}$. M y C. al que. por una razón u otra, le correspondió, por lo general. una mayor fijación real o aparente, de tal cation. $y$

- Crupo formado por la D. O y A o D. A y O, al que le correspondió todo lo contrario, $y$

(b) En cuanto a la crolución de la alcalinidad, [OH]

- Grupo formado por la D. M y N, y

- Grupo formado por el resto, encabezado normalmente por la $\mathrm{C}$ en razón de sus mayores contenidos de alcalinos antes mencionados.

8. Todos los cementos PUZ industriales han dado también resultado positivo en el ensayo de Frattini, a la edad de 7 o a la edad de 28 días habiendo apuntado además la posibilidad de que pueda haberse producido también en las fases liquidas del PU\%-4 y del PUZ-5. especialmente. cierta capacidad de cambio, al igual que la demostrada mediante los correspondientes cementos de mezcla preparados al efecto en el laboratorio para dicho fin. In ambos casos, de la elevada alcalinidad de sus fases líquidas, no se debe deducir. sin más. que los contenidos de alcalinos de la (s) puzolana (s) que los constituyen son también elevados. Ya que el aporte de los mismos puede haber sido proporcionado, en exclusiva o no. por la fracción de cemento Portland de cada uno de ellos. Lo que no es óbice para que la referida capacidad de cambio se origine en alguna medida.

\section{NoTA}

$\Lambda$ la vista de las conclusiones obtenidas. pero en especial la 1. es necesario recordar. una vez más. que desde el punto de vista de la construcción con hormigón de cemento. la única alcalinidad que posec interés tecnológico es la de origen portlandita. más no tanto phase alkalinity and fixation of $\mathrm{Ca}^{\circ}$ could be explained by the same reasons as put forvard for pozzolans

C and $M$.

7. According to the results of the Frattini test, the eight pozzolans studied, like the blended cements, can be classified into two groups depending on their pozzolanic activity; which is defined in terms of alkalinity: $\mathrm{OH}$, and fixation of $\mathrm{C}^{\prime} \mathrm{a}^{\prime}$. The latter property; in turn, apparently depends on the absolute $\mathrm{Al}_{2} \mathrm{O}_{3}(\%)$ concentration and/or relative amount of

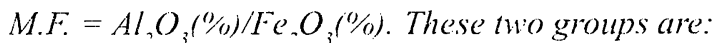

(a) In terms of the fixation of $\mathrm{Ca}^{2}$ or the evolution of $[\mathrm{CaO}]$ and concomitant erolution of [OH $]$ :

- Group comprising pozzolans $C V-10, C V-19, N, M$ and C, which for one reason or another, generally fixed larger quantities of this ion, and

- Group comprising D, O and A or D, A and $O$, which

fixed lesser quantities of $\mathrm{Ca}^{2}$, and

(b) In terms of evolution of allalinity, $[\mathrm{OH}]$ :

- Group comprising $D, M$ and $N$, and

- Group covering all the rest, normally headed by $C$ in view primarily of its higher alkali content, as discussed above.

7. All the indastrial POZ cements also showed positive Fratini test results in the 7-and 28-day trials. And, as in the laboratory-blended cements especially prepared for this purpose, some degree of exchange capacity. mal have been generated in the liquid phases of POZ-4 and especially POZ-5. In neither case, however should it be directly concluded from the high alkalinity of their. liquid phases that the alkali content of the pozalan(s) in their make-up is also high. Indeed, the source of this alkalinity may be solely from the Portland cement fraction. This does not, however preclude the possibility that some degree of exchange capacity is generated.

\section{NOTE}

In light of the above conclusions, particularly: conclusion 1, it must be stressed once again that from the standpoint of concrete construction, the only: alkalinity of practical interest is the alkalinity: from portlandite, whilst the alkalinity generated by alkaline 
por no decir ninguno, la promovida por elementos o iones puramente alcalinos tales como, por ejemplo, el $\mathrm{Na}, \mathrm{K}, \mathrm{Li}, \mathrm{Rb}$. etc., y el ión $\mathrm{NH}_{4}{ }^{\dagger}$. Por este razón, las investigaciones en torno aquélla, "deberán" ser siempre priorizadas para su aumento, mejora o preservación, simplemente, sobre las de ésta. elements such as $\mathrm{Na}, \mathrm{K}, \mathrm{Li}, \mathrm{Rb}$, or the cation $\mathrm{NH}_{4}$, has little, not to say no practical interest whatsoever: For this reason, research to expand, improve or simply maintain the former should take priority over research in the latter.

\section{BIBLIOGRAFIA}

(1) J. Sanz, A.Madani, J. M.Serratosa. J. S.Moya and S. Aza: "Aluminum-27 and Silicon-29 Magic-Angle Spinning Nuclear Magnetic Resonance Study of the Kaolinite-Mullite Transformation". J.Am.Ceram.Soc., 71 [10] C-418-C-42, 1988.

(2) R. Talero: "Contribución al Estudio Analitico y. Físico-Químico del Sistema:Cementos Puzolánicos-Yeso-Agua". Tesis Doctoral, Ftad. Ciencias Quimicas, Univ. Complutense de Madrid, 20 de noviembre de 1986.

(3) R.Talero: "Comparative XRD Ancilisis Ettringite Originating from Pozzolan and from Portland Cement".Cem. Concr. Res., Vol. 26, No. 8. pp. 1277-1283, 1996.

(4) R.Talero: "Kinetochemical and morphological differentiation of ettringites by the Le Chatelier-Ansttet test". Cem.Concr.Res. 32. 7(07-717, 2002.

(5) R. Talero: "Performance of Metakaolin and Pórtland Cements Forming Ettringite: Kinetic and Morphological Differences". 11 th Inter.. Congress on Chemestry of Cement, Vol. 2, pp. 853-867, 11-16 may 2003, DURBAN-Southafrica.

(6) R. Talero: "Kinctochemical and morphological differentiation of ettringites by metakaolin, portland cements and the Le ChatelierAnsttet test. Paramater: Vicat Needle Penetration". Silicates Industriels, Vol. 68, Nr.1-2, pp.1-10, 2003.

(7) R.Talero: "Comparative analysis of ettringite originating from metakaolin and from Portland cement by Le Chatelier-Ansttet test". Advances in Cement Research, Ref:: ACR 447 (made corrections for publication).

(8) R.Mejía: "Contribución al Análisis y Estudio del Sistema: Cementos Pórtland-Puzolanas y Escoria Siderírgica-Cloruros-Agua". Tesis Doctoral, Ftad. Ciencias Químicas, Univ. Complutense de Madrid, 26 de mayo de 1997.

(9) R. Lannegrand. G. Ramos y R. Talero: "Condition of knowledge about the Friedel's salt". Mater Construcc, Vol. 51, n" 262, págs. 63-71, abril/mayo/junio 200!.

(10) M. R. Jones. D. E. Mephee, J. A. Chudek, G. Hunter, R. Lannegrand, R. Talero and S.N. Scrimgeour: "Studies using '" Al MAS NMR of AFin and AFt phases and the formation of Friedel's salt". Cem.Concr. Res. 33, 177-182, 2003.

(11) H. F. W. Taylor: Cement Chemestry. 2nd. Edition. Published by Thomas Telford Publishing, Thomas Telford Services Ltd. 1 Heron Quay, London, E144JD. 1997.

(12) R. Talero: Patologías y Terapias Preventivas del Hormigón.II. La "Capacidad de Cambio" en las Adiciones Puzolánicas". Monografía n. 406 del Instituto de C. C. "Eduardo Torroja"-CSIC, Madrid, sept. 1991.

(13) Instrucción para la Recepción de Cementos RC-(04(R.D. 1797/2003 de 26 de diciembre, B.O.E. núm. 24 del 16 de enero de 2004. Secretaria General Téeniea, Servicio de Publicaciones, Ministerio de Fomento; Po de la Castellana, Madrid.

(14) Pliego de Prescripciones Téenicas Generales para la Recepeión de Cementos RC-75. Decreto de la Presidencia del Gobierno $1964: 1975$ de 23 de mayo (B.O.E. n²06 de 28 de agosto de 1975).

(15) ASTM C 114-94 Standard: "Standard Test Method for Chemical Analysis of Hydraulic Cement". Annual Book of ASTM Standards, Section 4 Construction, Vol. (04.()1 Cement; Lime;Gypsum, pp. 91-117, 1995.

(16) ASINEL: "Las Cenizas Volantesy y sus Aplicaciones". Selecciones Gráficas(Ediciones), Paseo de la Dirección, 52, MADRID.

(17) ASTM C $311-94$ b Standard: "Standard Test Method for Sampling and Testing Fly Ash or Natural Pozzolans for Use as a Admixture in Portland-Cement Concrete". Annual Book of ASTM Standars, Section 4 Construction, Vol. ()4.02. Concrete and Aggregates, pp. 187-193, 1995.

(18) Proyecto de Norma (INE 83.440: "Adiciones al hormigón. Cenizas Volantes: Determinación de álcalis solubles". AENOR. (19) ASTM C 595M-95 Standard: Standard Specification for Blended Hydraulic Cements. Annual Book of ASTM Standars, Section 4 Construction, Vol. 04.02, pp. 291-296, 1995.

(20) ASTM C 618-94 a Standard: "Standard Specificaction for Coal Fly Ash and Raw or Calcined Natural Pozzolan for Use as a Mineral Admixture in Portland Cement Concrete". Annual Book of ASTM Standards, Section 4 Construction, Vol. 04.02, pp. 304306,1995 .

(21) Pliego de Prescripciones para la Recepción de Conglomerantes Hidráulicos PCCH-64. Instituto de C. C. "Eduardo Torroja". (SIC. Normas y Manuales del IE Tec (Orden de 19 de abril de 1964, B.O.E. núm. 109, del 6 de amyo de 1964 y Corrección de Erratas aparecida en el B.O.E. núm. 222 del 15 de sept. 1964.

(22) ASTM ( 150-95 Standard: "Standard Specification for Portland Cement". Annual Book of ASTM Standards, Section 4 Construction, Vol. (04.01. pp. 128-132, 1995.

(23) R. Talero: "Sulphatic characterization of a Pozzolanic Addition: Accelerated Method of the Test to Determine it (Le ChatelierAnstet and ASTM C 4.52 tests)". AC $\triangle \wedge 10^{\text {th }}$ International Coal Ash Symposium, Established and Developing Coal Ash Uses Intern. Experiences, Section 8: Concrete I. Orlando - Florida - USA, january 17-23, 1993.

(24) V. Rahhal: "Caracterización de Adiciones Puzolánicas por Calorimetria de Conducción". Tesis Doctoral, E. T. S. de Ings. de ('aminos, (anales y Puertos, Dpto. de Ingeniería (Civil: Construcción, Univ. Politéenica de Madrid, 12 de diciembre de 2002.

(25) P. I.Nixon, (. L.Paget, R. Bollinnghaus and I. Canham: "The effect of Pfa with a high total alkali content on pore solution cmposition and alkali silica reaction". Magazine of Concrete Rescarch, Vol. 38, No.134, 1986. 
(26) V. Rahhal an R. Talero: "Effecet of three natural pozzolans in Portland cement hydration". Mater Construcc, Vol. 53, n" 269, pp.29-40, 2003.

(27) V. Rahhal y R. Talero: "Hydration of Portland cements: calorimetric influence of two fly ashes". Journal of Thermal Analysis and Calorimetry (accepted for publiaction).

(28) V. Rahhal y R. Talero: "Influencia de las adiciones aluminicas sobre la calorimetria de los cementos Portland". Asociación Téenica de Tecnologia del Hormigón. XV Reunión Téenica. Seminario de Hormigones Especiales, Universidad Técnológica Nacional. Facultad Regional de Santa Fe, Argentina, 21 al 24 de octubre de 2003.

(29) Norma UNE-EN 450): "Cenizas iolantes como adición al hormigón. Definiciones, especificaciones y control de calidad". AENOR. Calle Génova n" 6. 280(0)4-MADRID.

(30) Intorme privado n"18.261 del IETec. 23 de julio de 2003.

(31) R. Mcía, S. Delvasto. (" Gutiérre\% and R. Talero: "Chloride diffiusion measured permeability test in normal and blended cements". Adv. Cem. Res. 15, No. 3, July, 113-118. 2003.

(32) R. Talero: "Qualitative Analysis of Natural Pozzolans, Fly Ashes and Blast Furnace Slags by XRD". Journal of Materials in Civil Engincering, Vol.2, No.2. pp.10)-115, 1990).

(33) R. Talero. M R. Bollati and F. Hernándes-O.: "Manufacturing of mortars and concretes non-traditional byPortland cement. metakaolin and gypsum (15,05\%)". Mater Construcc. Vol. 49. No 256, pp. 29-41, 1999.

(34) R. Talero: "El ahorro de energia en la fabricación de cemento: L'lltimos ar'ances sobre las adiciones puzolánicas silicicas". Informes de la Construcción. Vol. $38 . n^{\circ} 385$, noviembre 1986.

(35) R. Talero: "Uso y aplicaciones del humo de silice en la construcción". Cimbra. Revista de la Ingeniería Téenica de Obras Públicass, año XXX. número 298, pp. 36-40, abril-may'o 1994.

(36) M. R. Bollati, R. Talero and F. Hernánder-O.: "Manufacturing dosage of concrete with silica fume whose compressive strength is > 40 Kp'cm at lery early ages (5-10 hours)". Mater Construce, Vol. 48, n² 252, pp. 61-66,1998.

(37) Instrucción de Hormigón Estructural EHE (R.D. 2661/1998, de 11 de diciembre).Secretaria General Téenica. Servicio de Publicaciones. Ministerio de Fomento, Po de la Castellana. Madrid.

(38) Guía de Aplicación de la Instrucción de Hormigón Estructural. Fdificación. Secretaría General Téenica, Servicio de Publicaciones, Ministerio de Fomento. I" Ed. oct.2(0)3.

\section{FE DE I:RRATAS}

Fn el Número 275, correspondiente a los meses de julio/agosto/septiembre de 2004 y respecto al trabajo "Caracterización físicoquímica de los morteros romanos de El Ruedo (Almedinilla. Córdoba)/Physical-chemical characterization of roman mortars in E1 Ruedo at Almedinilla, Córdoba", se ha deslizado un crror de impresión en lo que respecta a la fïgura 5 del mismo, adjuntándose la definitiva.

Asimismo, indicar que el e-mail del autor de contacto, es el siguiente: umlferog(a uco.es
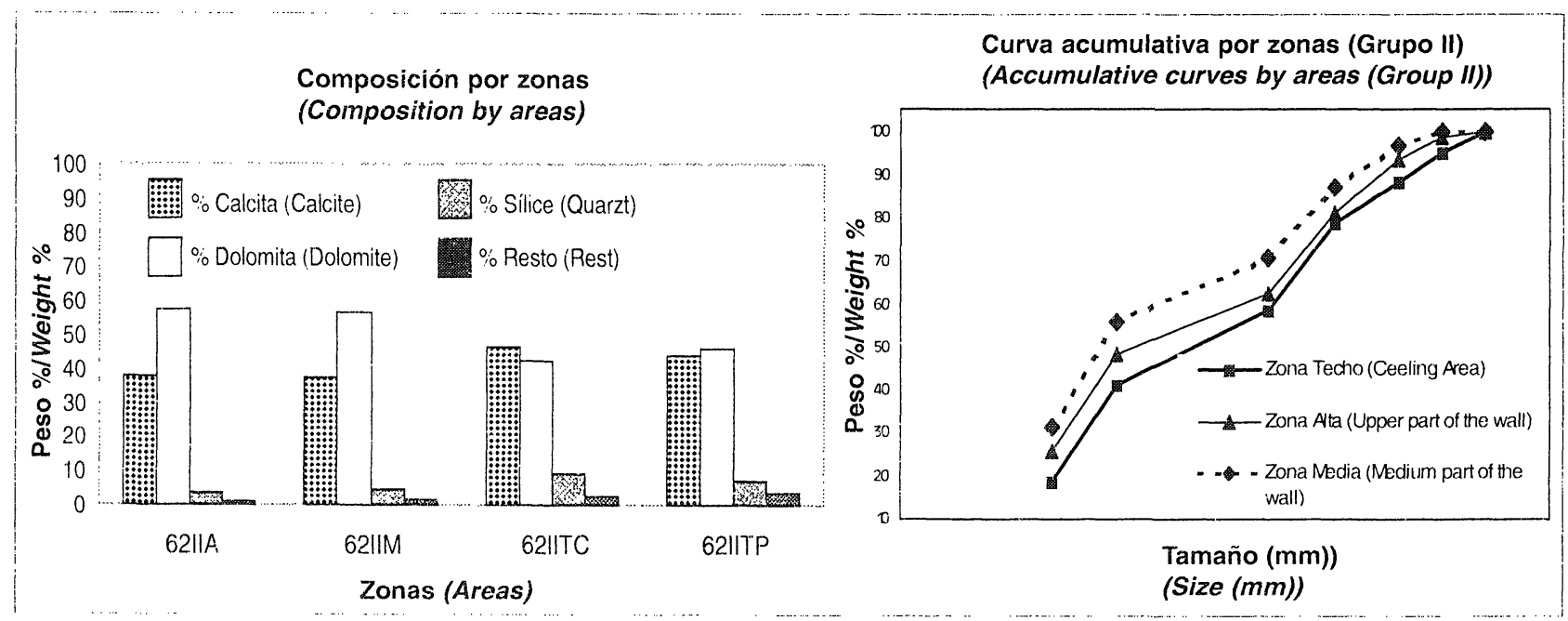

Figura 5.- (omposición del grupo Il, de la estancia 62, asi como curva granulométrica acumulada.

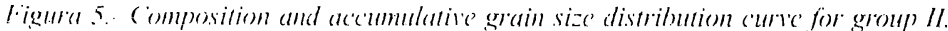

